\title{
KNIGHT SHIFT AND QUADRUPOLE INTERACTION \\ IN SINGLE CRYSTAL MAGNESIUM
}

\author{
by \\ PATRICK DANIEL DOUGAN \\ B.Sc. (Hons.), University of British Columbia, 1966
}

A THESIS SUBMITTED IN PARTIAL FULFILMENT OF

THE REQUIREMENTS FOR THE DEGREE OF

\author{
MASTER OF SCIENCE \\ in the Department \\ of
}

Physics

We accept this thesis as conforming to the required standard

THE UNIVERSITY OF BRITISH COLUMBIA

April, 1969 
In presenting this thesis in partial fulfilment of the requirements for an advanced degree at the University of British Columbia, I agree that the Library shall make it freely available for reference and Study. 1 further agree that permission for extensive copying of this thesis for scholarly purposes may be granted by the Head of my Department or by his representatives. It is understood that copying or publication of this thesis for financial gain shall not be allowed without my written permission.

Department of

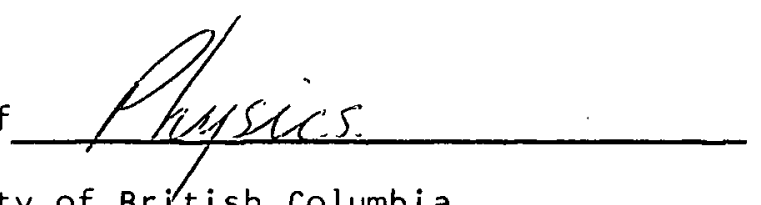

The University of British Columbia Vancouver 8 , Canada

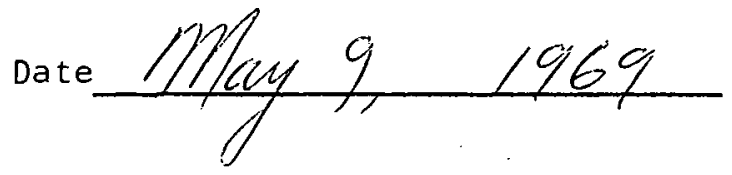




\section{ABSTRACT}

The nuclear magnetic resonance in single crystal magnesium has been studied at $4.2^{\mathrm{O}} \mathrm{K}$. The isotropic Knight shift, the anisotropic Knight shift and the quadrupole interaction have been investigated. The values for the parameters measured are $\mathrm{K}=(0.1127 \pm 0.0005) \%$, $\mathrm{K}^{\prime}=(0.0004 \pm 0.0002) \%, \mathrm{e}^{2} \mathrm{qQ} / \mathrm{h}=324 \pm 6 \mathrm{KHz}$. These results are compared with those of Rowland in powdered magnesium to reveal the Knight shift is independent of temperature. This contrasts directly with the behavior of cadmium. The quadrupole interaction is shown to increase by $40 \%$ as temperature is lowered to $4.2^{\circ} \mathrm{K}$ from room temperature. 
LIST OF ILLUSTRATIONS . . iii

ACKNOWLEDGEMENTS ... ' iv

CHAPTER

I INTRODUCTION . . I

II REVIEW OF THE THEORY

A. Knight Shift 3

1. The Isotropic Shift 4

2. Anisotropic Knight Shift 6

B. Quadrupole Interaction 7

1. The Quadrupole Moment 9

2. The Electric Field Gradient 11

3. Perturbation Calculations 12

C. Field Gradient of the Ionic Model 19

III APPARATUS AND TECHNIQUE

A. General Description 22

B. Crystal vs. Powder 24

C. Experimental Technique and Considerations 25

1. The Sample . 25

2. Modulation and Detection 27

3. The Magnetic Field 29

IV RESULTS AND DISCUSSION

A. Experimental Observations . 34

B. Comparison with Powder Data 38

C. Role of the Conduction Electrons 41

$\mathrm{V}$ CONCLUSIONS

A. Comparison with Cadmium 44

B. Further Studies 46

BIBLIOGRAPHY 


\section{LIST OF ILLUSTRATIONS}

FIGURE

Page

$1 \quad \mathrm{x}, \mathrm{z}, \mathrm{X}$ and $\mathrm{z}$ Axes

2 Quadrupole Splitting of the Magnetic Resonance of a Nucleus Spin $3 / 2$

3 Angular Dependencies of theSplittings of the Satellites of the Central Line

4. Schematic Diagram of the Spectrometer

5 Special Four-Samplé Probe for Field Homogenization

6 Oscilloscope Traces for Probe in Inhomogeneous and

31 Homogeneous Fields

7 Resonance Line Corresponding to the $\left(\frac{1}{2}<-\frac{1}{2}\right)$ Transition of $\mathrm{Mg}^{25}$ in a Single Crystal

8 Measured Frequency Shift of Central Component of the $\mathrm{Mg}^{25}$ Resonance

9 Theoretical Powdex Signal Shapes 


\section{ACKNOWLEDGEMENTS}

With pleasure I thank Dr. D. LI. Williams for his help, instruction and patience with this work, as well as for his sponsorship and support in obtaining financial assistance.

For his assistance in all parts of this work, I thank Dr. S. N. Sharma who also constructed the spectrometer used.

I am grateful to Dr. A. Akhtar for the loan of the crystal and for his help with its preparation.

For financial support, my thanks go to the National Research Council and the Dean's Committee on Research. 


\section{CHAPTER I}

\section{INTRODUCTION}

Since nuclei have magnetic moments, they are sensitive to magnetic fièlds originating in the spin and orbital currents of the electrons. Lacking an electric dipole moment; nuclei are insensitive to homogeneous electric fields but since some may possess electric quadrupole moments, they can experience torques in sufficiently inhomogeneous electric fields produced in part by the electron clouds. Since these interactions couple the nuclear spin system to the electron system, the study of nuclear magnetic resonance can yield valuable information on the electronic properties.

If a magnetic field $\mathrm{H}_{0}^{\circ}$ is applied in the $\mathrm{z}$ direction to a system of $\mathrm{N}$ nuclei and $\mathrm{n}$ conduction electrons, the resulting Hamiltonian, neglecting nuclear dipolar and exchange interactions, can be written $(1)$ :

$$
\begin{aligned}
H= & H_{e}+\sum_{i=1}^{n} \beta H_{o}\left(L_{z}^{(i)}+2 S_{z}^{(i)}\right)+H_{n} \\
& +\sum_{j=1}^{N} g \mu_{o} H_{o} I_{z}^{(j)}+\sum_{i=1}^{n} \sum_{j=1}^{N} H_{e n}(i j)
\end{aligned}
$$

where $\mathrm{H}_{e}$ is the Hamiltonian for the electrons in the field of the ion cores; $\mathrm{H}_{\mathrm{n}}$ represents the Hamiltonian for the ion cores. The terms containing $\mathrm{H}_{0}$ are the Zeeman energies of the electrons and nuclei. $H_{e n}$, the Hamiltonian for the noncoulombic interaction between the nuclear spin and the electron orbital and spin coordinates 
can be approximated by including only the magnetic dipole and electric quadrupole parts of the interaction,

$H_{e n}=2 \beta \gamma \bar{\hbar} \bar{I} \cdot\left[\frac{\bar{I}}{r^{3}}-\frac{\bar{s}}{r^{3}}+\frac{3 \bar{r}(\bar{s} \cdot \bar{r})}{r^{5}}+\frac{8}{3} \pi \cdot \bar{s} \delta(\bar{r})\right]+\frac{1}{6} \sum_{i j} V_{i j} Q_{i j}$

The Knight shift and quadrupole coupling, to be discussed in chapter two, are the most marked manifestations of these interactions.

Studies in cadmium $(2,3,4)$ have revealed a large temperature variation of the Knight shift which has not yet been satisfactorily explained. The speculation that this behavior might: be characteristic of other divalent hexagonal close-packed metals, notably magnesium and zinc, motivated this study of the nuclear magnetic resonance of magnesium. Because of the several advantages outlined in chaptex three, this study was performed on a single crystal; before this, the resonance had been observed in magnesium metal only in a powdered specimen.

The investigation of the Knight shift was complicated by the presence of a quadrupole moment of the magnesium nucleus. Consequently, a determination of the quadrupolar interaction was also achieved. 
CHAPTER II

REVIEW. OF THE THEORY

\section{A. Knight Shift}

The magnetic field seen by a nuclear spin in a resonance experiment on a metal has, in addition to the applied external field, contributions from the surrounding nuclei and electrons. This field is almost always higher than that which the same nucleus would experience in a non-metallic substance. The resulting shift in field is called the "Knight shift", which is qualitatively defined as

$$
\mathrm{K}=\frac{\Delta H}{H}
$$

where $\mathrm{H}$ is the external applied field and $\mathrm{H}$ is the additional field due to the hyperfine coupling. Of course, this change in field causes a corresponding shift in resonance frequency, so where a constant fjeld is employed, $\mathrm{K}$ can be measured by determining .

$$
\mathrm{K}=\frac{\nu_{\mathrm{m}}-\nu_{\mathrm{R}}}{\nu_{\mathrm{R}}}
$$

where $\nu_{\mathrm{m}}$ and $\boldsymbol{V}_{\boldsymbol{R}}$ are the resonance frequencies in the metal and a non-metallic reference respectively. Similar shifts in non-metallic compounds, called chemical shifts, although much smaller, are present and necessitate specifying the reference compound when quoting Knight shift values.

The origin of the Knight shift lies in the magnetic interaction part of the Hamiltonian (eqn. 1): 


$$
H_{1}=2 \beta \gamma \hbar \overline{\mathrm{I}} \cdot\left[\frac{\bar{l}}{r^{3}}-\frac{\bar{s}}{r^{3}}+\frac{3 \bar{r}(\bar{s} \cdot \bar{r})}{r^{5}}+\frac{8}{3} \pi \bar{s} \delta(\bar{r})\right]
$$

Assuming for simplicity that the electron orbital momentum is quenched, the expectation value of hyperfine coupling for a given nuclear spin can be written

$$
\gamma=\overline{\mathrm{I}} \cdot \sum\left\langle\phi_{k}\left|2 \beta\left\{\frac{3\left(\bar{r}_{k} \cdot \bar{s}_{k}\right) \bar{r}_{k}}{r_{k}^{5}}-\frac{\bar{s}_{k}}{r_{k}{ }^{5}}+\frac{8 \pi}{3} \bar{s}_{k} \delta\left(\bar{r}_{k}\right)\right\}\right| \phi_{k}\right\rangle_{F}
$$

Since orbits with two paired electrons cannot contribute to the coupling, the summation is over the unfilled orbits near the top of the Fermi distribution. Here $\bar{x}_{k}$ and $\bar{s}_{k}$ are the position and spin of the unpaired electron in the orbit

1. The Isotropic Shift.

If the symmetry of the electron environment of the nuclear spin is cubic or higher, only the $\delta$-function coupling will be nonzero. Since this term arises from the contact of the s-state electron wave function with the nucleus at $r=0$, we need only consider $\phi_{k}(0)$. In this case the coupling (6) becomes (5)

$$
-\gamma \hbar(\overline{\mathrm{I}} \cdot \overline{\mathrm{H}}) \frac{8 \pi}{3}\left\langle\left|\phi_{k}(0)\right| 2\right\rangle_{E_{F}} x_{e}^{s}
$$

where $x_{e}{ }^{s}$ is the total spin susceptibility of the electrons.

This is entirely equivalent to the interaction of the nuclear spin with an extra magnetic field

$$
\Delta H=\frac{8 \pi}{3}\left\langle\left|\phi_{k}(0)\right| 2\right\rangle_{E_{\mathrm{F}}} x_{e}^{s} H_{0}
$$


This term is independent of the orientation of the applied field and in its fractional form, $\Delta \mathrm{H} / \mathrm{H}_{\mathrm{O}}$, is referred to as $\mathrm{K}_{\text {iso }}$, the isotropic Knight shift. The corresponding resonance frequency shift gives

$$
\mathrm{K}_{\text {iso }}=\frac{\Delta \mathrm{H}}{\mathrm{H}_{\mathrm{o}}}=\frac{\Delta \nu}{\nu_{\mathrm{R}}}=\frac{8 \pi}{3} \mathrm{x}_{\mathrm{e}}^{\mathrm{s}}\left\langle\left|\phi_{\mathrm{k}}(0)\right|^{2}\right\rangle_{\mathrm{E}_{\mathrm{F}}}
$$

Effects which have been ignored above but which also contribute to the shift are core polarization and orbital paramagnetism. Core polarization, an important effect in the transition elements, arises from an unpaired outer electron interacting with the s-electrons in the closed-shell ion core of the atom. This interaction produces perturbations on the core-electron wave functions which depend on whether the spin of the core-electron is parallel or anti-parallel to the spin of the conduction electron. Because of the contact of the s-electron and the nucleus, a net magnetic field results from the perturbed electron spin density at the nuclear site. This is an important effect in the transition metals.

The presence of an applied magnetic field modifies the occupied energy levels of the system, mixing in higher unoccupied states having a net orbital magnetic moment in the direction of the field. This "unquenching" of the electron orbital paramagnetism is responsible for the chemical shifts which are also present in the diamagnetic compounds used for reference. For simple metals, these effects are much smaller than the Knight shift. 


\section{Anisotropic Knight Shift}

Where the symmetry of the electron environment of the nuclear spin is lower than cubic, the Knight. shift is complicated by the inclusion of a term which is dependent on the orientation of the crystal with respect to the applied field.

One contribution to the anistropy arises from the magnetic dipole term of the interaction Hamiltonian (eqn. 2)

$$
H_{d}=2 \beta \gamma \hbar \bar{I} \cdot\left[\frac{\bar{s}}{r^{3}}-\frac{3 \bar{r}(\bar{s} \cdot \bar{x})}{r^{5}}\right]
$$

As was done for the isotropic shift, the expectation value of this term is summed over the unfilled electron orbitals near the top of the Fermi distribution:

$$
2 \beta \gamma \overline{\bar{I}} . \quad \sum_{k}\left\langle\phi_{k}\left|\frac{\bar{s}}{x^{3}}-\frac{3 \bar{r}(\bar{s} \cdot \bar{r})}{r^{5}}\right| \phi_{n}\right\rangle_{F}
$$

As in the previous case, this effect can be intexpreted as an additional field $\Delta \mathrm{H}_{\text {anis }}$ experienced by the nucleus where; taking the $\mathrm{z}$ component,

$$
\Delta H_{\text {anis }}=2 \beta \hbar \sum_{k}\left\langle\left.\phi_{k}\right|_{r} ^{s} \frac{z}{3}-\frac{3 z(\bar{s} \cdot \bar{r})}{r^{5}} \mid \phi_{k}\right\rangle_{F}
$$

Here also we define a fractional shift $\Delta \mathrm{H} / \mathrm{H}$ and label it $\mathrm{K}$ anis Carrying out this summation for the case in which the crystal has axial symmetry, Bloembergen and Rowland ${ }^{(6)}$ arrive at the expresșion in spherical co-ordinates,

$$
\mathrm{K}_{\text {anis }}=\frac{\Delta \nu_{\text {anis }}}{\nu_{R}}=\frac{\Delta H_{\text {anis }}}{H_{0}}=\beta^{2} V_{0} N\left(E_{F}\right) q_{F}\left(3 \cos ^{2} \theta-1\right)
$$


where $\theta$ is the angle between the crystal symmetry axis and the direction of the applied field, $V_{\mathrm{O}}$ is the atomic volume and $N\left(E_{\mathrm{F}}\right)$ is the number of electron states near the Fermi surface. Here

$$
q_{F}=\left\langle\psi\left|3 \cos ^{2} \phi-1\right| \psi\right\rangle_{F}
$$

is the quadrupole moment of the electrons near the Fermi surface where $\psi$ is the average wave function and $\phi$ the angle of the radius vector from the symmetry axis. The quadrupole moment can be determined in part by the crystal structure and apparently accounts for the sign of $K^{\prime}$ which may be positive or negative.

A further contribution may come from the anisotropy of the magnetic susceptibility which in some cases, as in scandium, ${ }^{(7)}$ ranges up to $30 \%$. This effect has the same field and angular dependence as the dipolar effect making them phenomenologically indistinguishable. Since all the mechanisms of the Knight shift anisotropy are not properly understood, their total effect is generally grouped in the single parameter

$$
\frac{\Delta H_{\text {anis }}}{H_{\mathrm{o}}}=\mathrm{K}_{\text {anis }}=\frac{1}{3}\left(\mathrm{~K}_{\|}-\mathrm{K}_{\perp}\right)\left(3 \cos ^{2} \theta-1\right)
$$

$\mathrm{K}_{\|}$and $\mathrm{K}_{\perp}$ are the shifts corresponding to $\theta=0^{\circ}$ and $\theta=90^{\circ}$ respectively. With the quantity $\frac{1}{3}\left(K_{\|}-K_{\perp}\right)$ denoted by $K^{1} / 2$, the total Knight shift can be conveniently expressed as

$$
\frac{\Delta \nu}{\nu_{\mathrm{R}}}=\frac{\Delta \mathrm{H}}{\mathrm{H}_{\mathrm{o}}}=\mathrm{K}_{\text {iso }}+\frac{\mathrm{K}^{\prime}}{2}\left(3 \cos ^{2} \theta-1\right)
$$

B. Quadrupole Interaction

Nuclei are unaffected by homogeneous electric fields because they lack a dipole moment. However, they do have quadrupole electric 
moments which will interact with inhomogeneous electric fields and manifest themselves through coupling with the electron system and the nuclear spin system.

A direct method of deriving this interaction is to consider the electrostatic interaction energy of a nucleus of charge distribution $\rho(\bar{x})$ with a potential $V(\bar{x})$ due to its surrounding environment of charged particles,

$$
E=\int \rho(\bar{x}) V(\bar{x}) d^{3} \bar{x}
$$

We can expand $V(x)$ in a Taylor series about the origin.

$v(x)=v(0)+\sum_{i} x_{i}\left(\frac{\partial v(\bar{x})}{\partial x_{i}}\right)_{r=0}+\frac{1}{2} \sum_{i, j}\left(\frac{\partial v(\bar{x})}{\partial x_{i} \partial x_{j}}\right)_{r=0}+\ldots$

and define $v_{i}=\left(\frac{\partial v(\bar{x})}{\partial x_{i}}\right)_{r=0} \quad, \quad v_{i j}=\left(\frac{\partial v(x)}{\partial x_{i} \partial x_{j}}\right)_{r=0}$

to get

$$
E=V(0) \int \rho(\bar{x}) d^{3} x+\sum_{i} v_{i} \int x_{i} \rho(\bar{x}) d^{3} \bar{x}+\frac{1}{2} \sum_{i j} v_{i \cdot j} \int x_{i} x_{j} \rho(\bar{x}) d^{3} \bar{x}+\ldots
$$

The first term is the electrostatic energy of the nuclear point charge taken at the center of mass of the nucleus. Since nuclear states have definite parity, the second term, involving the electric dipole of the nucleus, $\int x_{i} \rho(\bar{x}) d^{3} \bar{x},:$ vanishes for symmetry reasons. (8) The third term is the electric quadrupole term. We can always find principle axes of the potential $V(\bar{x})$ such that $V_{i j}=0$ if $i \neq j$. Here any electronic charge which lies within the nucleus has been neglected since, if we consider atoms, only s-electrons penetrate the nucleus and having spherical symmetry, give: no quadrupolar coupling. 
Terms higher than the quadrupole are ignored since either they vanish for symmetry reasons or their effect is negligibly small.

1. The Quadrupole Moment

By defining a quantity $Q_{i j}=\int\left[3 x_{i} x_{j}-\delta_{i j} r^{2}\right] \rho(\bar{x}) d^{3} \vec{x}$ to get $\int x_{i} x_{j} \rho(\bar{x}) d^{3} \bar{x}=\frac{1}{3}\left[Q_{i j}+\int \delta_{i j} r^{2} \rho(\bar{x}) d^{3} \bar{x}\right]$, we can write for the quadrupole interaction energy

$$
\begin{aligned}
E & =\frac{1}{2} \sum_{i j} v_{i j} \int x_{i} x_{j} d^{3} \bar{x} \\
& =\frac{1}{6} \sum_{i j}\left[v_{i j} Q_{i j}+v_{i j} \delta_{i j} \int r^{2} \rho(\bar{x}) d^{3} \bar{x}\right]
\end{aligned}
$$

Since the potential $V(\bar{x})$ must satisfy LaPlace's equation at the origin, the second term of eqn. (8) vanishes, leaving

$$
E=\frac{1}{6} \sum_{i j} V_{i j} Q_{i j}
$$

Replacing the classical density $\rho$ by its operator, we get añ Hamiltonian for the quadrupole interaction

$$
H_{Q}=\frac{1}{6} \sum_{i j} V_{i j} Q_{i j}
$$

where $Q_{i j}$ is an operator over the protons in the nucleus,

$$
Q_{i j}=e \sum_{k}\left(3 x_{i k j k} x_{j k}-\delta_{i j k} x^{2}\right)
$$

In the resonance experiment we are interested only in the ground state of a nucleus and since the separation in energy between ground and excited states is very large compared to the magnitude of 
$\mathrm{H}_{\mathrm{Q}}$, for a perturbation calculation involving $\mathrm{H}_{\mathrm{Q}}$ we need knowledge only of matrix elements of the form $\left\langle I m\left|Q_{i j}\right| \mathrm{Im}\right\rangle$. These elements are, except for $\mathrm{m}$, diagonal in all quantum numbers such as the spin I, which characterize the nuclear ground state. Here $m$ is the magnetic quantum number corresponding to an orientation of the nuclear spin $I$. By means of the Wigner-Eckart Theorem, we can express the matrix elements of $Q_{i j}$ as elements of an equivalent operator in angular momentum space. The theorem yields

$$
\left\langle I^{\prime}\left|Q_{i j}\right| I m\right\rangle=c\left\langle I^{\prime}\left|\frac{3}{2}\left(I_{i} I_{j}+I_{j} I_{i}\right)-\delta_{i j} \bar{I}^{2}\right| I m\right\rangle
$$

where $\mathrm{C}$ is a constant independent of $\mathrm{m}$ or $\mathrm{m}^{\prime}$. The constant $\mathrm{C}$ is related to the quantity called "the nuclear electric quadrupole moment", denoted by the letter $Q$ and usually measured in units of charge $e . Q$ is defined by

$$
\mathrm{eQ}=\left\langle\left.\mathrm{II}\right|_{\mathrm{Q}_{33}} \mid \mathrm{II}\right\rangle
$$

as the expectation value of $Q_{33}$ in the state in which the component of $I$ along the $\mathrm{z}$ axis is a maximum $(m=I)$. By writing this in terms of 8 )

$$
e Q=\int \rho_{\text {II }}(\bar{x})\left(3 z^{2}-r^{2}\right) d^{3} \bar{x}
$$

we see that it gives us a measure of the asymmetry of the nuclear charge distribution. $Q$ has the units of area and is commonly expressed in barns $\left(10^{-24} \mathrm{~cm}^{2}\right)$. 
The constant $C$ can now be evaluated in terms of $e Q$

$$
\begin{gathered}
e Q=C\left\langle I I\left|3 I_{z}^{2}-\bar{I}^{2}\right| I I\right\rangle=C\left[3 I^{2}-I(I+I)\right] \\
C=\frac{e Q}{I(2 I-I)}
\end{gathered}
$$

We therefore have a final expression for our quadrupole Hamiltonian:

$$
H_{Q}=\frac{1}{6} \sum_{i j} V_{i j} Q_{i j}=\frac{e Q}{6 I(2 I-I)} \sum_{i j} V_{i j}\left[\frac{3}{2}\left(I_{i} I_{j}+I_{j} I_{i}\right)-\delta_{i j} I^{2}\right]
$$

In order that the nucleus have a quadrupole moment, the spin must be $I \geq 1$. This is apparent semiclassically, for if the spin were $\frac{1}{2}$ and the nuclear charge distribution were spheroidal in shape with the spin axis being the spheroid's axis of symmetry, the two possible spin orientations would correspond to the same effective energy. A nucleus with $I=0$ is, of course, spherically symmetric and has no preferred orientation at all.

\section{The Flectric Field Gradient}

The magnitude of the electric field gradient at the nucleus can be described by the tensor $V_{i j}$ introduced earlier. This tensor is symmetric (curl $E=0$ ) and traceless $\left(\nabla^{2} V=0\right.$ at the origin) so it has, in general, five independent components. These can be reduced to three by taking the principle axis $\left(X, Y, Z\right.$ ) as a basis so $V_{i j}$ can be expressed in diagonal form. The equation $\nabla^{2} \mathrm{~V}=\mathrm{V}_{\mathrm{XX}}+\mathrm{V}_{\mathrm{YY}}+\mathrm{V}_{\mathrm{ZZ}}=0$ must still be satisfied so it is sufficient to define two symbols $\eta$ and $q$, called the asymmetry parameter and the field gradient, by 


$$
\begin{aligned}
& \text { eq }=v_{\mathrm{ZZ}}=\left(\frac{\partial^{2} \mathrm{~V}}{\partial \mathrm{Z}^{2}}\right)_{\mathrm{o}} \\
& \eta=\frac{\mathrm{v}_{\mathrm{XX}}-\mathrm{v}_{\mathrm{YY}}}{\mathrm{v}_{\mathrm{ZZ}}}
\end{aligned}
$$

The Hamiltonian (29) can now be written:

$$
H_{Q}=\frac{e^{2} q Q}{4 I(2 I-1)}\left[3 I_{z}^{2}-I(I+I)+\frac{\eta}{2}\left(I_{+}^{2}+I_{-}^{2}\right)\right]
$$

where

$$
I_{ \pm}=I_{x} \pm i I_{y} .
$$

If in the magnesium system we assume axial symmetry and take the axis to be in the $z$ direction so that $\eta=0$ ( since $V_{X X}=V_{Y Y}$ ), we arrive at our final form,

$$
H_{Q}=\frac{e^{2} q Q}{4 I(2 I-I)}\left[3 I_{z}^{2}-I(I+I)\right]
$$

3. Perturbation Calculations

In some cases, the nuclear quadrupole interaction is so great it is responsible for the entire dependence of the energy of a nucleus on its spin orientation. In these cases, resonance experiments in zero or very low fields are possible. In these "low-field" studies the Zeeman splitting can often be treated as a perturbation on the quadrupole energy. We shall be dealing here with the "high-field" studies where the quadrupole interaction is small enough to be treated as a perturbation on the magnetic energies. 
The application of a.magnetic field to a spin system gives rise to the Zeeman energies $H_{M}=-g \beta \bar{H} . \bar{I}$. With $z \| \vec{H}$, the applied field, a coordinate system is defined in which $\mathrm{H}_{M}$ is diagonal and has the eigenvalues

$$
\bar{E}_{\mathrm{m}}^{(o)}=-\mathrm{g} \beta \mathrm{Hm} \equiv-\mathrm{h} \nu_{\mathrm{L}} \mathrm{m} \quad, \mathrm{m}=-\mathrm{I},-\mathrm{I}+\mathrm{I}, \ldots, \mathrm{I}
$$

- There are thus $2 \mathrm{I}+\mathrm{I}$ equally spaced magnetic energy levels, separated by $h \nu_{L}$, between which dipole transitionscan be induced by the application of a radio-frequency field tranşerse to $\overline{\mathrm{H}}$. The selection rules $\Delta m= \pm I$ give rise to a single resonance line at the Larmor frequencies (figure $2 \mathrm{a}$ ).

In general the axes $\mathrm{z}$ and $\mathrm{z}$ for the magnetic and quadrupole interaction differ. We have then for our Hamiltonian describing the two effects,

$$
\mathrm{H}=\mathrm{H}_{\mathrm{M}}+\mathrm{H}_{\mathrm{Q}}=-\gamma \mathrm{hH}_{\mathrm{O}} \mathrm{I}_{\mathrm{z}}+\frac{\mathrm{e}^{2} \mathrm{qQ}}{4 \mathrm{I}(2 \mathrm{I}-\mathrm{I})}\left[3 \mathrm{I}_{\mathrm{Z}}^{2}-\mathrm{I}(\mathrm{I}+\mathrm{I})\right]
$$

If the quadrupole coupling is weak compared to the magnetic interaction, we consider the spin quantized along the $z$ axis. Defining the $x$ axis to lie in the plane of $z$ and $z$ we have (fig. I)

$$
I_{\mathrm{Z}}=I_{\mathrm{z}} \cos \theta+I_{\mathrm{x}} \sin \theta
$$

In equation (35), we now have

$$
\begin{aligned}
H= & -\gamma \hbar_{0} I_{z}+\frac{e^{2} q Q}{4 I(2 I-I)}\left[3 I_{z}^{2} \cos ^{2} \theta+3 I_{x}^{2} \sin ^{2} \theta\right. \\
& \left.+3\left(I_{z} I_{x}+I_{x} I_{z}\right) \sin \theta \cos \theta-I^{2}\right]
\end{aligned}
$$




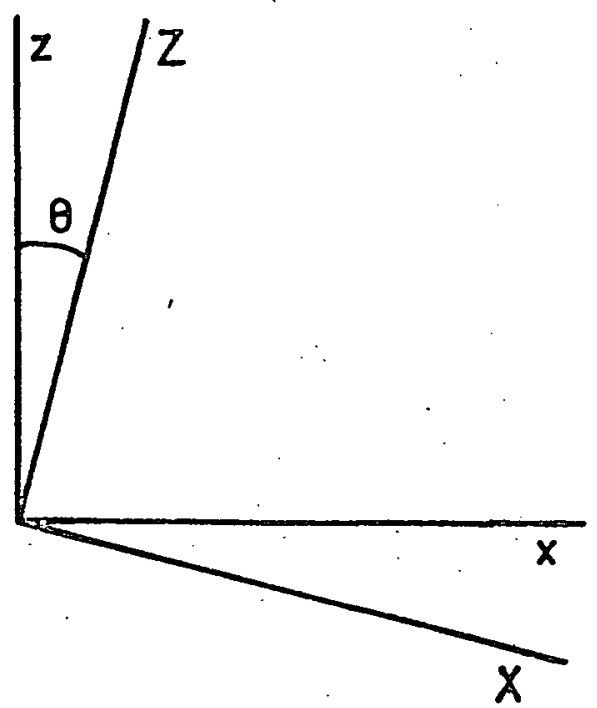

Fig. $1 \mathrm{x}, \mathrm{z}, \mathrm{X}$ and $\mathrm{Z}$ axes

By writing $I_{x}=\frac{I}{2}\left(I^{+}+I^{-}\right)$and $I_{y}=(I / 2 i)\left(I^{+}-I^{-}\right)$, eq. (37) gives (9)

$$
H=-\gamma \bar{h}_{0} I_{z}+\frac{e^{2} \cdot q Q}{4 I(2 I-I)}\left[\begin{array}{c}
\frac{1}{2}\left(3 \cos ^{2} \theta-I\right)\left(3 I_{z}^{2}-I(I+I)\right. \\
+\frac{3}{2} \sin \theta \cos \theta\left[I_{z}\left(I^{+}+I^{-}\right)+\left(I^{+}+I^{-}\right) I_{z}\right.
\end{array}\right]
$$

This is written to show up the diagonal $(\Delta m=0)$ and offdiagonal $(\Delta \mathrm{m}= \pm 1$, +2$)$ matrix elements of $\mathrm{H}_{\mathrm{Q}}$ which will come into the pexturbation treatment.

The total Hamiltonian $\mathrm{H}=\mathrm{H}_{\mathrm{M}}+\mathrm{H}_{\mathrm{Q}}$ has energy levels

$$
E_{m}=E_{m}^{(0)}+E_{m}^{(1)}+E_{m}^{(2)}+\ldots
$$

where $E_{m}(p)$ is the contribution to the energy of the perturbation of order . 
The zero-th order term, that of the Zeeman coupling alone is $\operatorname{Em}^{(0)}=-\gamma \hbar H \mathrm{Hm}=-h \nu_{L} \mathrm{~m}$.

The first order term Em ${ }^{(1)}=\left\langle\mathrm{m}\left|\mathrm{H}_{\mathrm{Q}}\right| \mathrm{m}\right\rangle$ is

$$
E m^{(1)}=\frac{I}{4} h \nu_{Q}\left(3 \mu^{2}-1\right)\left(m-\frac{1}{3} a\right)
$$

where for convenience the following terms have been introduced:

$$
\nu_{Q}=\frac{3 \mathrm{e}^{2} \mathrm{qQ}}{\mathrm{h} 2 \mathrm{I}(2 \mathrm{I}-1)}, \mathrm{a}=\mathrm{I}(\mathrm{I}+\mathrm{I}), \mu=\cos , \quad \nu_{\mathrm{L}}=\frac{\gamma \mathrm{H}}{2 \pi}
$$

If the perturbation is carried to second order, we get

$$
E_{m}^{(2)}=\sum_{n \neq m} \frac{\left|\left\langle n\left|H_{0}\right| m\right\rangle\right|^{2}}{E^{(0)}{ }_{-E n}^{(0)}}=-h\left(\frac{\nu_{Q}^{2}}{2 \nu_{L}}\right) m\left[\begin{array}{l}
\frac{3}{2}{ }^{2}\left(1-\mu^{2}\right)\left(8 m^{2}-4 a+1\right) \\
+\frac{3}{8}\left(1-\mu^{2}\right)^{2}\left(-2 m^{2}+2 a+1\right)
\end{array}\right]
$$

Because of these changes in the energy levels, there are now several resonance frequencies:

$$
\nu_{m}=\frac{E_{m-1}-E_{m}}{h}=\nu_{L}+\nu_{m}(1)+\nu_{m}(2)+\ldots
$$

In first order, the magnetic resonance line is split into $2 \mathrm{I}$ components with relative intensity nearly equal to $I(I+1)-m(m-I)$ for the $m \rightarrow m-1$ transition. For systems with half-integral spins, the central $\left(-\frac{1}{2} \rightarrow \frac{1}{2}\right)$ component is unaffected and the shifted components, called satellites are arranged symmetrically about the central transition in pairs (figure $2 \mathrm{~b}$ ). The frequency of the splitting is given by

$$
\nu_{m}^{(1)}=\frac{E_{m-1}^{(I)}-E_{m}^{(I)}}{h}=\nu_{Q}\left(m-\frac{1}{2}\right) \frac{3 \mu^{2}-1}{2} .
$$

which vanishes for $\mathrm{m}=\frac{1}{2}$. The $\mathrm{Mg}^{25}$. isotope, with a nuclear spin $\mathrm{I}=5 / 2$, would have its resonance. Iine split into five components. 

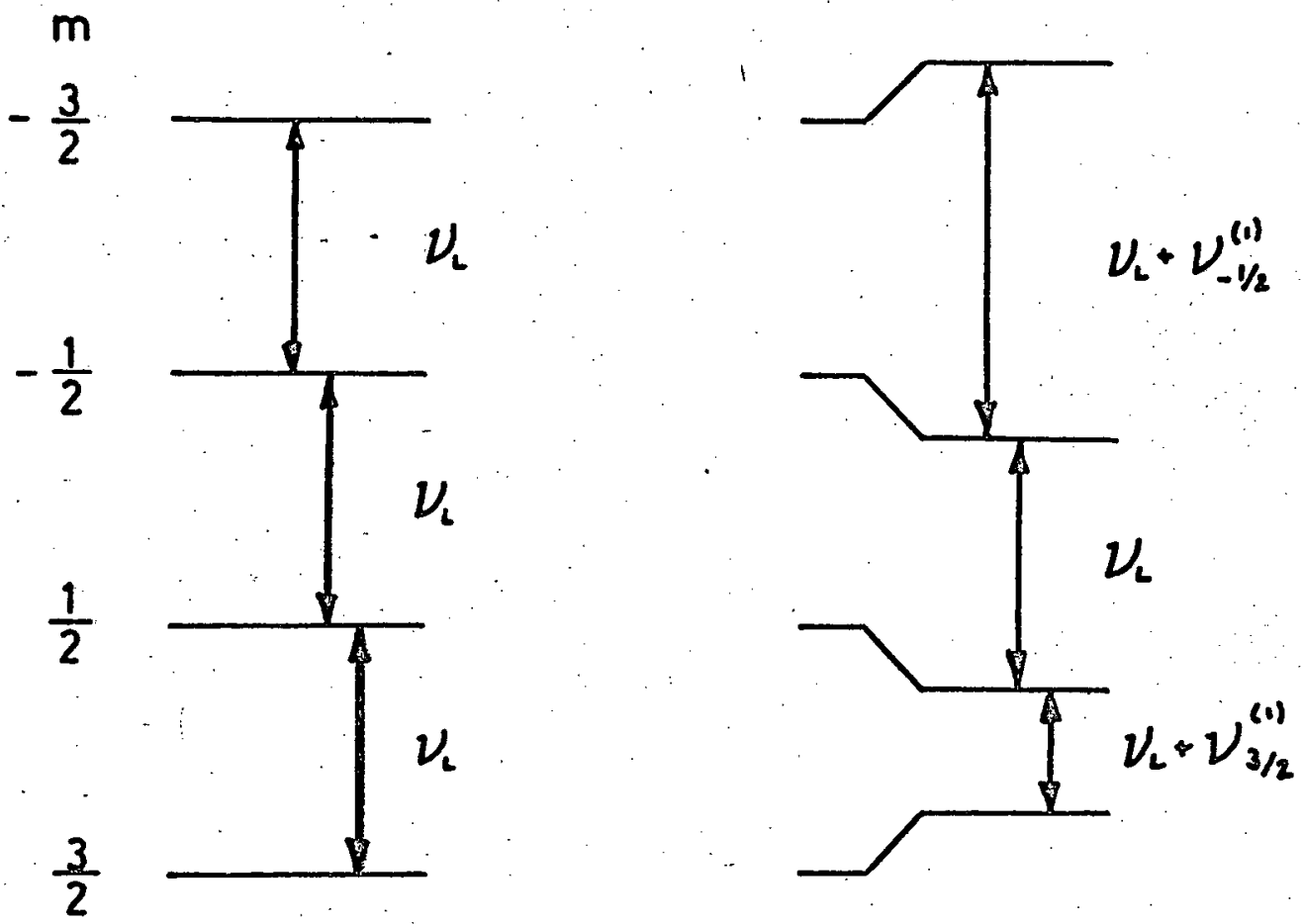

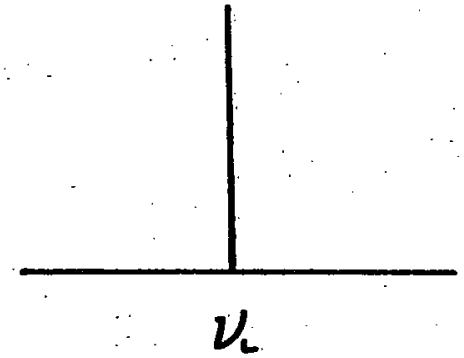

$a$

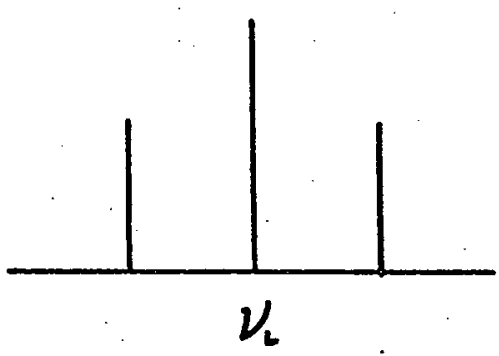

b

Fig. 2 Quadrupole Splitting of the Magnetic Resonance of a Nucleus Spin $3 / 2$ a: Applied Field only

$\mathrm{b}$ : With Quadrupole Interaction

The energy levels shown at top in units of h. The bottom shows the line shape for a polycrystalline sample. 
If the perturbation is carried to second order the central component is no longer undeviated but is shifted by

$$
\nu_{\frac{1}{2}}(2)=\frac{E_{-\frac{1}{2}}^{(2)}-E_{\frac{1}{2}}(2)}{h}=\frac{-\nu_{Q}^{2}}{16 V_{L}}\left(a-\frac{3}{4}\right)\left(1-\mu^{2}\right)\left(9 \mu^{2}-1\right)
$$

The other components are of course shifted as well, but will not be considered here. The angular dependencies of equations (4I) and (42) are shown in figure 3 .

In a powdered or polycrystalline sample the random distribution of orientation of the crystal axis with respect to the magnetic field gives rise to a continuous distribution of frequencies. The effect then of the quadrupole interaction in splitting the resonance line into satellites or in shifting the central line (second-order effect) is manifested as a broadening of the line. As a result of this, the interaction must be weak to be observable:

Because of the advantages outlined in chapter four, the experiment may be performed on a single crystal where $\mu=\cos \theta$ is well defined. If the crystal is rotated about an axis perpendicular to the magnetic field, a plot of the resonance frequency as a function of angle of rotation will give the curves of figure 3 . The first (figure 3 a) shows the frequency interval between the central line and the satellites for the first order. shifts while the second (figure 3,b) demonstrates the second-order shift of the central component.

The satellite lines are very sensitive to the effects of strains and imperfections $(10)$ in the crystal and are thus considerably wider than the central component and correspondingly more 


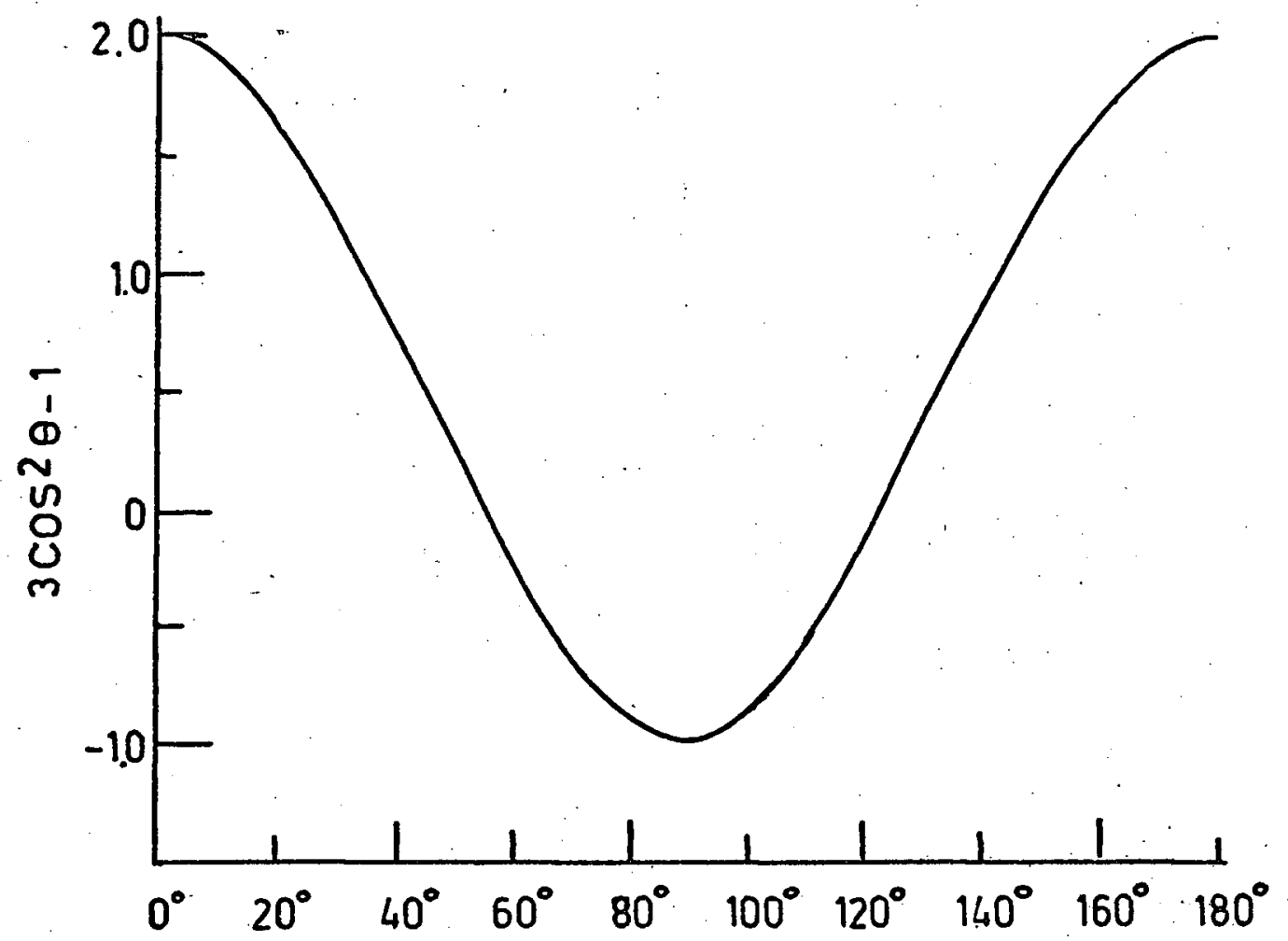

Fig. 3a The angular dependence of the Knight shift anisotropy and/or the satellite lines to first order

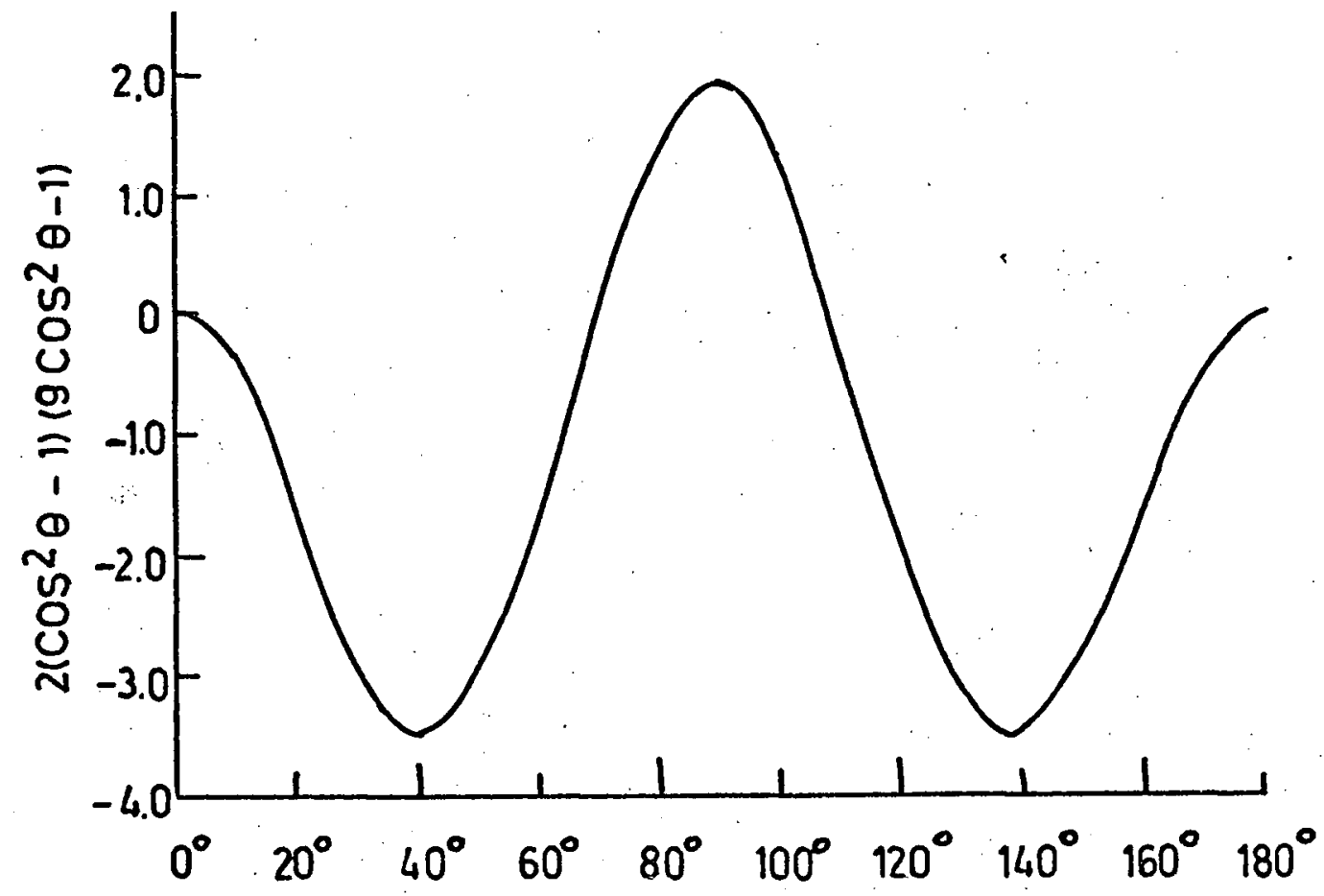

Fig. 3b The angular dependence of the shift of the central $\left(\frac{1}{2}-\frac{1}{2}\right)$ transition due to the quadrupole interaction 
difficult to observe or measure accurately. The parameter of intere̊st, $\mathrm{eq}^{2} \mathrm{Q} / \mathrm{h}$, commonly called the quadrupole coupling constant, can therefore in some cases, be more accurately determined from measurement of the shift of the central component.

\section{C.' Field Gradient of the Ionic Model}

As discussed earlier, the potential $\mathrm{V}$ producing the field gradient arises from external charges of other nuclei or electrons. A charge $e$ at the point $x, y, z$ of the principle axes produces at the origin a field gradient of

$$
\text { eq }=\frac{\partial^{2} v}{\partial z^{2}}=e \frac{\left(3 z^{2}-r^{2}\right)}{r^{5}}
$$

or in terms of spherical coordinates

$$
q_{i}=\frac{\left(3 \cos ^{2} \phi_{-1}\right)}{r^{3}}
$$

If the ionic crystal exists as an array of non-overlapping, spherically symmetric ions, then the resulting local electric field gradient, $\nabla_{i j}$, would be that arising from all the other ions. This could be determined by summing the above $q_{i}$ over all lattice sites a distance $\bar{r}_{i}=\left(x_{i}, y_{i}, z_{i}\right)$ from the origin to get.

$$
q_{\text {latt }}=\sum_{i} \frac{3 z_{i}{ }^{2}-r_{i}{ }^{2}}{r_{i}^{5}}
$$

However, the ion is distorted both by the quadrupolar field of the nucleus and by the local electric field gradient itself. Because of 
their $I / r^{3}$ dependence, the electrons in the closed shell of the ion make a substantial contribution to the total field gradient. This additional contribution is denoted by $\gamma_{\infty} v_{i j}$ where $\gamma_{\infty}$ is termed the "antishielding factor".

This induced quadrupolar coupling is the sum of two terms:

a) the nuclear quadrupole moment (N.Q.M.) polarizes the ionic shell and produces an electronic quadrupole moment which can then interact with the external gradient $V_{i j}$, and

b) the external gradient also polarizes the ionic shell which produces an additional gradient at the nucleus which will then interact with the N.Q.M.

Formally the effects are treated as the interaction of a total field $\left(I+\gamma_{\infty}\right) V_{i j}$ with the nuclear moment $Q_{i j}$.

Physically, the antishielding is due to a rearrangement of electronic charge. If $Q$ is positive, the potential energy is a minimum along the axis of $Q$ and the electrons undergo an angular redistribution and concentrate there. This "angular excitation" is due to excitement of s-electrons into higher d states and of p-electrons into higher $f$ states. Accompanying this may be a radial shift of charge inward along the axis and outward at right angles to it. This "radial excitation", resulting from the excitation of p-electrons into higher $\mathrm{p}$ states and $\mathrm{d}$ electrons into higher $\mathrm{d}$ states, tends to reinforce the effect of the N.Q.M. The angular redistribution will cause shielding while the radial excitation is responsible for antishielding. The 
antishielding effect is usually relatively larger where present, since the radial redistribution should propagate to the outer regions of the ion. The antishielding factor for small ions can be positive or negative depending on the electron distribution of the core. For heavier ions, $\gamma_{\infty}$ is always large and positive.

Effectively then, the ionic contribution to the electric field gradient is denoted

$$
q_{\text {ion }}=z\left(1+\gamma_{\infty}\right) q_{\text {lattice }}
$$

where $\mathrm{Z}$ is the normal valence of the ion. The effects of the valence electrons has been excluded here. That they can have a substantial effect will be discussed in chapter four. 


\section{CHAPTER III}

\section{APPARATUS AND TECHNIQUE.}

Efforts here were directed at detecting the steady-state resonance signal to determine the frequency dependence as the magnetic field orientation was varied with respect to a particular crystal axis. The experimental apparatus and techniques used were those most commonly employed in nuclear magnetic resonance spectroscopy.

\section{A. General Description}

In this case the steady-state resonance was detected by modulating the magnetic field and sweeping the frequency of the applied r.f. field. The angular oxientation of the sample with respect to the magnetic field could be varied by rotating the magnet about the sample which was rigidly connected to a frame above the magnet.

A coil wound about the crystal sample formed the inductive load of the tank circuit of a modified Pound-Knight-Watkins oscillating detector. The sample was held within copper bomb rigidly fixed to a stainless steel coaxial line leading to the oscillator. A liquid helium cryostat enclosed the bomb and coaxial line. The output of the oscillator was fed into a lock-in amplifier and the signal recorded on a strip-chart recorder. An electronically produced sawtooth waveform was applied to a voltage variable capacitance diode which was incorporated into the tank circuit of the oscillator to provide the frequency sweep. A schematic diagram of the apparatus is shown in figure 4. 


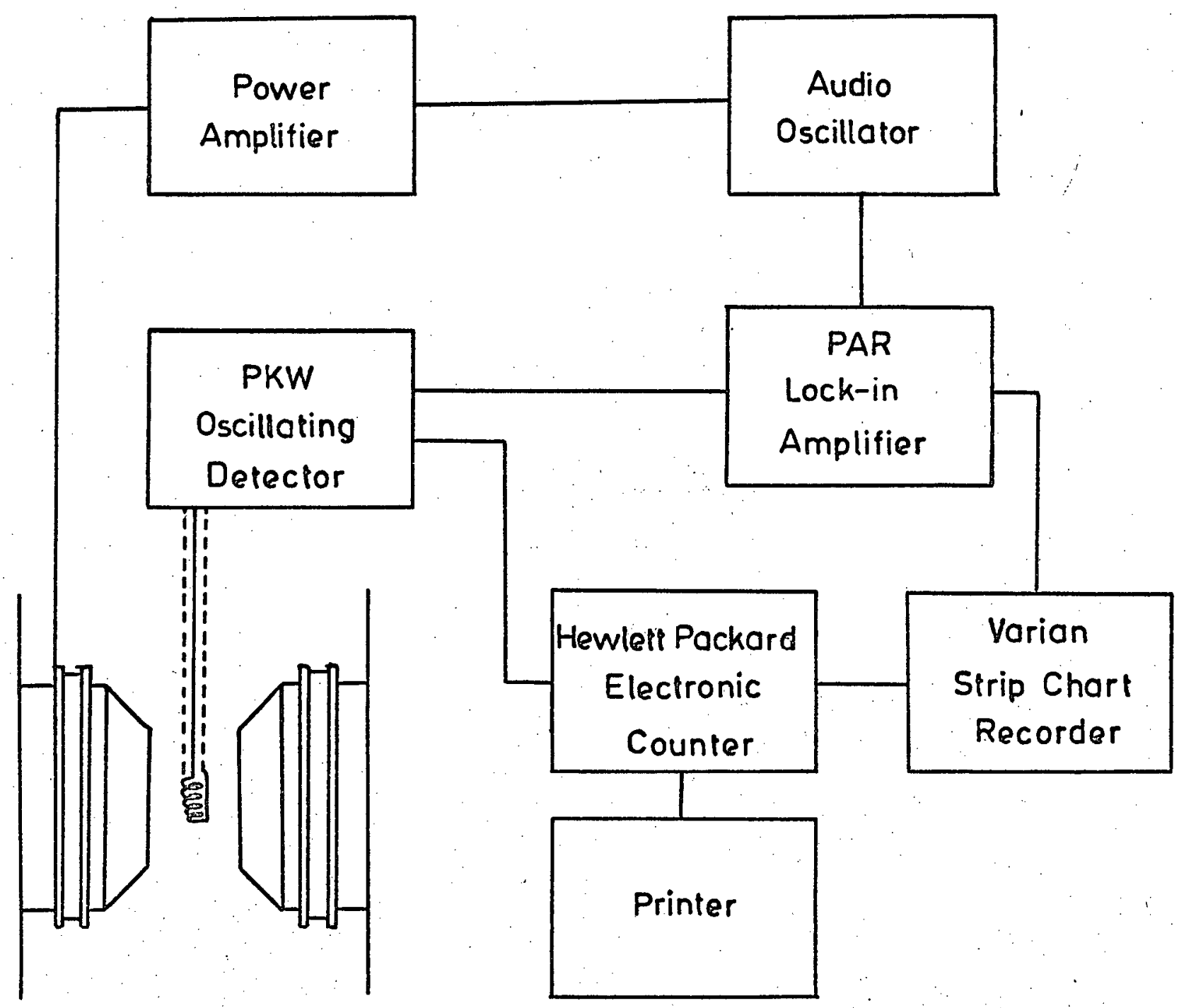




\section{B. Crystal vs. Powder}

The study of n.m. $r$. in metals is more complex than in non-conductors due to the ordinary conductive losses in the sample. The sample can be penetrated by the $r . f$. field only a distance given by the classical skin depth $\delta=\frac{1}{2 \pi} \sqrt{\frac{\rho}{\nu x}}$ where $\rho$ is the resistivity, $\boldsymbol{\nu}$ the frequency, and $\boldsymbol{x}$ the magnetic permeability. This "skin effect" causes not only attenuation of the r.f. field as it enters the metal, but also a phase shift between the surface and points within the sample. To overcome this problem, the sample is usually prepared in the form of a powder, wire or foil, having at least one dimension small compared with the skin depth. If this condition is not satisfied, not only does the attenuation cause a reduction in intensity, but the phase shift causes a distortion of the observed resonance absorption which can be described as a mixing of the absorption and dispersion modes of the complex nuclear susceptibility. The effects on the line shape of this mixing is understood ${ }^{(13)}$ and can be corrected for if necessary. At low temperatures, a pure sample of a good conductor may pass into the "anomalous skin effect" region where the mean free path of the electrons is large compared to the normal skin depth: This condition facilitates greater penetration of the r.f. field.

Experiments on single crystals are then more difficult because of the low sensitivity afforded by the skin effect, so most work on metals is performed in powdered samples. This method has several advantages. The line is much more intense because essentially all the sample is exposed to the r.f. field. Separating the modes is no longer necessary since the observed signal will be purely absorptive. 
Since the sample is made up of randomly oriented crystallites, several difficulties also arise. The line is necessarily broadened due to the variation of resonance position as a function of crystal orientation. When the effect under study is orientation dependent, such as the Knight shift anisotropy or the quadrupole effects, the observed line is asymmetricatly broadened, the result of a weighted averaging of the individual particle orientation. These lines can be interpreted (14) to give meaningful results but this method becomes quite complex when two or more angularly dependent effects are operating together (Chapter four).

With a single crystal sample, the resonance frequency can be observed to change as the angle between the magnetic field and the crystal axis is varied. In this way, very small effects can be observed which escape detection in the powdered technique. Using a single crystal also allows the measurement of bulk material properties and not just surface effects.

\section{Experimental Technique and Considerations}

\section{The Sample}

The sample itself was a cylindrical single crystal 1.5 inches long by 0.5 inches in diameter. It was spark cut from a larger ingot: of $99.999 \%$ pure magnesium so that the cylindrical axis made a right angle with the $\langle 0001\rangle$ axis. Since magnesium is hexagonal, the ellectric field gradient is considered to be axially symmetric $(\eta=0)$ about the hexagonal $\langle 0001\rangle$ axis of the crystal. Thus when 
the crystal was mounted with its cylindrical axis vertical, a horizontal rotation of the magnet produced the required angular variation from $0^{\circ}$ to $360^{\circ}$.

$\mathrm{X}$-ray analysis determined the accuracy of the crystal. orientation to within one degree. After spark-cutting and slight annealing, the crystal was lightly etched in a $10 \%$ solution of HCl to reduce surface strains and imperfections.

The nature of the coil was determined by the operating characteristics of the oscillator. Since the optimum sensitivity seemed to require a small capacitance in the tank circuit which could be varied over a very limited range, the frequency of oscillation was determined by the inductance of the sample coil. Trial and error produced a $2 \mathrm{~cm}$ long cylindrical coil consisting of 450 turns of \#44 copper wire wound on the crystal over a single layer of $0.0005^{\prime \prime}$ Mylar. Too long a coil would result in the sample occupying a large volume in the magnetic field, possibly leading to inhomogeneity; too short a coil would decrease the number of nuclei available and reduce the signal.

The single layer of Mylar was incorporated to protect the crystal surface from the coil windings and to slightly increase the inductance so the coil length could be kept down. However, the Mylar increased the quality factor, $Q$, of the circuit slightly more than was desirable, decreasing the filling factor and thus the sensitivity. The filling factor, the fraction of the total radio frequency magnetic 
energy that is stored in the sample, should be kept as high as possible for maximum signal.

2. Modulation and Detection

The oscillating detector was a Pound-Knight-Watkins type, built and modified by S.N. Sharma. A full description of it may be found in his thesis. (15)

An increase in sensitivity was attained by use of phasesensitive detection, a common and necessary technique for weak signal detection. In this, the field is modulated so that the signal appears at the input of the detector as a variation in amplitude of a periodic function

$$
s(t)=c \sin (w t+\delta)
$$

where is the phase difference between the signal and reference, $R(t)$, with which the signal is mixed. For simplicity assume that the reference is sinusoidal, $R(t)=A$ sin wt. After mixing, the result is integrated by passing through a low-pass filter of integrating time $\mathrm{T}$ to give an output

$$
\begin{aligned}
E_{0} & =\frac{C}{T} A \int_{\tau}^{\tau+T} \sin w t \sin (w t+\delta) d t \\
& =\frac{C A \cos \delta}{2}
\end{aligned}
$$

so that maximum signal is attained when reference and signal are in phase or $180^{\circ}$ out of phase $\left(\delta=0^{\circ}, 180^{\circ}\right)$. In practise the device behaves as a very narrow banded amplifier. When the reference signal is at the frequency of the modulation, the detected output is 
proportional to the first derivative of the absorption signal when the modulation is sufficiently small. (16)

The oscillator frequency rather than the magnetic field was swept to determine the resonance frequency. This was necessitated by the high magnetoresistance encountered in the magnesium specimen. It was found that sweeping the field caused variations in resistance of the sample, thus affecting the Q of the tuned circuit. This caused large oscillating drifts of the r.f. level which could not be contained on the chart recorder without reducing amplification and making signal detection impossible.

The modulation can also be applied to either the $r . f$. frequency or the magnetic field. Frequency modulation is difficult because the $x . f$. level of the tank circuit is frequency dependent; a periodic oscillation of the r.f. level appears which would, of course, not be filtered by phase-sensitive detection since it is of the same frequency as the signal. At the input of the detector, instead of the function of equation (27) would be

$$
V(t)=c \sin \left(w t+\delta_{1}\right)+D \sin \left(w t+\delta_{2}\right)
$$

where $c \sin \left(w t+\delta_{1}\right)$ is the signal and $D \sin \left(w t+\delta_{2}\right)$ is the effect of modulation picked up on the r.f. level. Here $\delta_{1}$ and $\delta_{2}$ are phase differences from the reference and are not in general equal. After mixing and filtering, the detected result is

$$
E_{1}=\frac{C A \cos \delta 1}{2}+\frac{D A \cos \delta_{2}}{2}
$$


Thus the modulation pickup is manifested as a d.c. drift which may be too large to balance in the detector or may overload the lock-in amplifier making it necessary to operate at a lower amplification. Unfortunately, the high magnetoresistance caused the same effect on the r.f. level of the tank circuit when modulation was applied to the magnetic field.

The d.c. drift caused by this modulation pickup was overcome by detecting the second harmonic of the resonance signal. The signal appearing at the output is not purely sinusoidal and can be Fourier decomposed into terms of higher harmonics. It can be shown that when modulation of sufficiently small amplitude is applied, the output of the phase sensitive detector detecting at the $\mathrm{n}^{\text {th }}$ harmonic of the modulation is proportional to the $n^{\text {th }}$ derivative of the resonance line shape ${ }^{(16)}$. Since the modulation pickup has a lower harmonic content than the signal, the system can be used at higher sensitivity and the problem of d.c. drifts sidestepped. The modulation applied to the field was one gauss peak-to-peak amplitude at $20 \mathrm{~Hz}$. The second derivative was detected by using a reference of $40 \mathrm{~Hz}$ in the lock-in.

Because the signal amplitude is dependent on the modulation amplitude, large modulations may be applied to increase the signalto-noise ratio. This causes distortion broadening of the line which, however, can be corrected for ${ }^{(16)}$ if necessary.

3. The Magnetic Field

The experiment was performed in a magnetic field of 20 
Kilogauss. The choice of field was dictated by several factors. The PKW oscillator performed sensitively over a range of about 8 to $25 \mathrm{MHz}$, but with some alterations and proper choice of sample coil could be made to perform adequately at $5 \mathrm{MHz}$. In a static field of $20 \mathrm{Kg}$ the magnetic moment of magnesium would produce resonance at a frequency of $5.2 \mathrm{MHz}$. This relatively high field was advantageous since the signal is proportional to $\mathrm{H}^{2}$. Although the high field magnet available would provide up to $24 \mathrm{Kg}$, its homogeneity decreased drastically at high fields, putting an upper limit on the field available. The magnet, a rotatable Magnion model, equipped with a field sensing device and a current regulator, supplied a very stable field (less than one gauss variation) over a period of days. In the centre of the pole faces were adjustable cores, called $\mu$-shims, used to optimize the homogeneity of the field. Without a high degree of homogeneity, the nuclei in various parts of the sample expexience different magnetic fields and the observed resonance line is broadened. The required homogeneity, about one part in $10^{5}$, was achieved by using a special probe consisting of four vials of lithium chloride solution arranged symmetrically about the centre of the gap in the plane of the pole faces (fig. 5). The pole faces were adjusted to produce the same field at each of the four samples; this was determined when the resonant peaks from each coincided (fig. 6). The $\mu$-shims were used as a fine adjustment to yield a homogeneity of about one part in $10^{5}$ in a region $3 \mathrm{~cm}$ in diameter. 


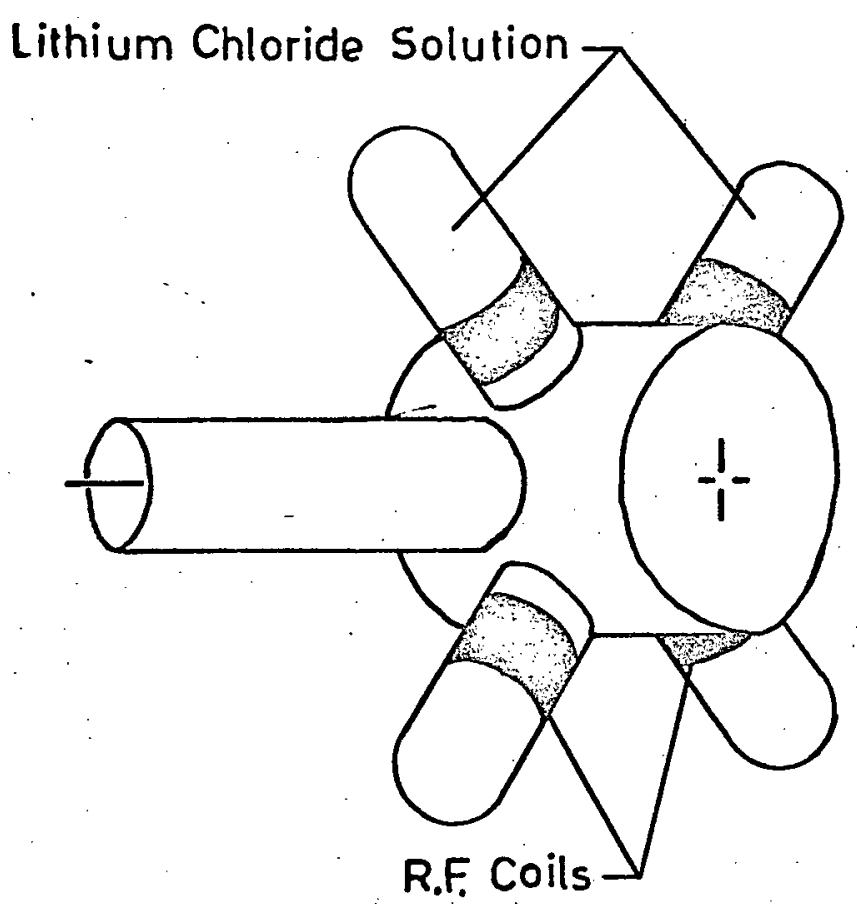

Fig. 5 Special Four-Sample Probe for Field Homogenization

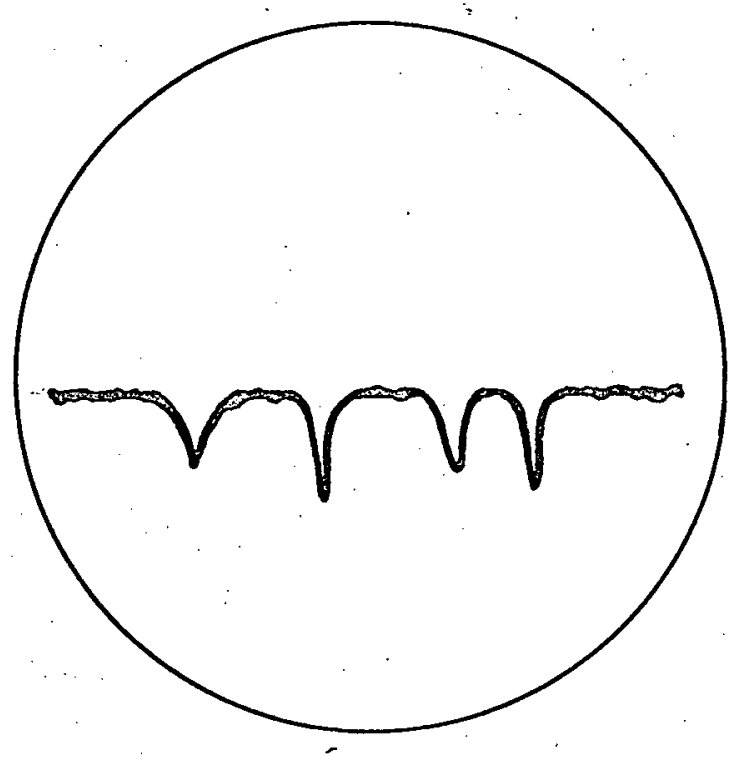

Fig. 6a 0scilloscope trace showing signal when probe in inhomogeneous field

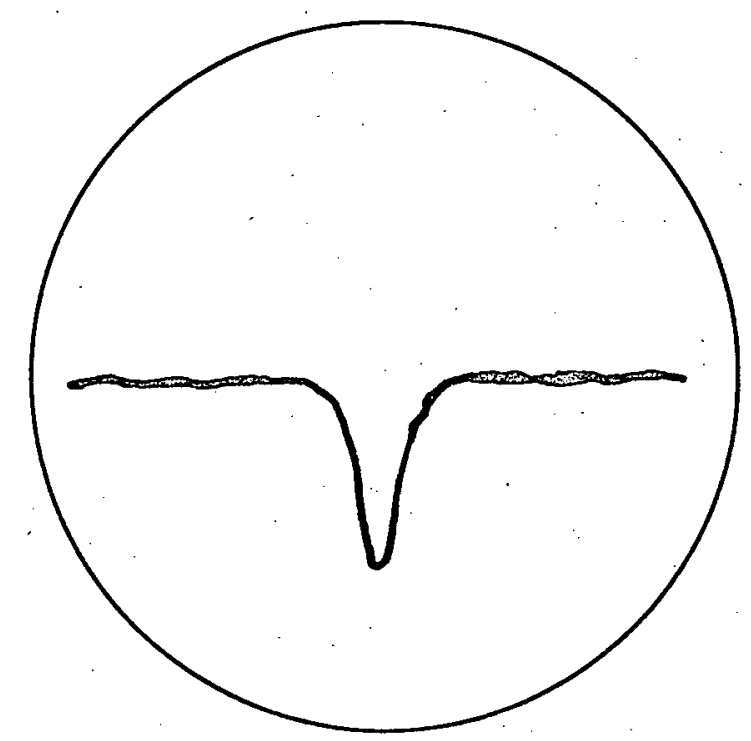

Fig. 6b Field homogenized 
The field was measured before and after the experiment by the use of a deuterium oxide probe the same size as the sample and in the exact position the sample was to occupy.

Further enhancement of the signal was achieved by performing the observations at liquid helium temperature, $4.2^{\circ} \mathrm{K}$, since the available nuclear magnetization varies inversely with temperature. The low temperature system was of a standard design; it consisted of an inner liquid helium dewar, connected to a vacuum system and contained within an outer liquid nitrogen dewar. 


\section{CHAPTER IV}

\section{RESULTS AND DISCUSSION}

\section{A. Experimental Observations}

For reasons discussed earlier, frequency. measurements of the central $\left(-\frac{1}{2} \rightarrow \frac{1}{2}\right)$ transition of the magnesium single crystal were taken as the direction of the magnetic field was varied. The effects of orientation on this frequency as the magnetic field is rotated in the plane perpendicular to the $\langle 0001\rangle$ axis is given by combining equations (16) and (42):

$$
\begin{aligned}
\nu_{\frac{1}{2}}= & \nu_{0}+\nu_{0}\left[K_{\text {iso }}+\frac{K^{\prime}}{2}\left(3 \cos ^{2} \theta-I\right)\right] \\
& +\frac{\nu_{Q}^{2}}{16 \nu_{0}}\left[I(I-I)-\frac{3}{4}\left(\cos ^{2} \theta-I\right)\left(9 \cos ^{2} \theta-I\right)\right]
\end{aligned}
$$

where $\nu_{R}$ the reference frequency and $\nu_{L}$ are taken to be equal and denoted $\nu_{0}$.

Here we have added together the expressions for the separate interactions of the anisotropic Knight shift (first order) and the nuclear quadrupole interaction (second order). This is justified since the higher order terms are negligible and the contribution resulting from interference terms of the two interactions vanishes identicaliy. (14) The measureable parameters from the observation are $\mathrm{K}_{\text {iso }}$ and $\mathrm{K}^{\prime}$, the Knight shift parameters, and the magnitude of the quadrupole coupling constant, $\mathrm{e}^{2} \mathrm{q} / \mathrm{h}$.

The resonance line of figure 7 was observed at $4.2^{\circ} \mathrm{K}$. Since at this temperature the line was observed only at low $r . f$. fields 


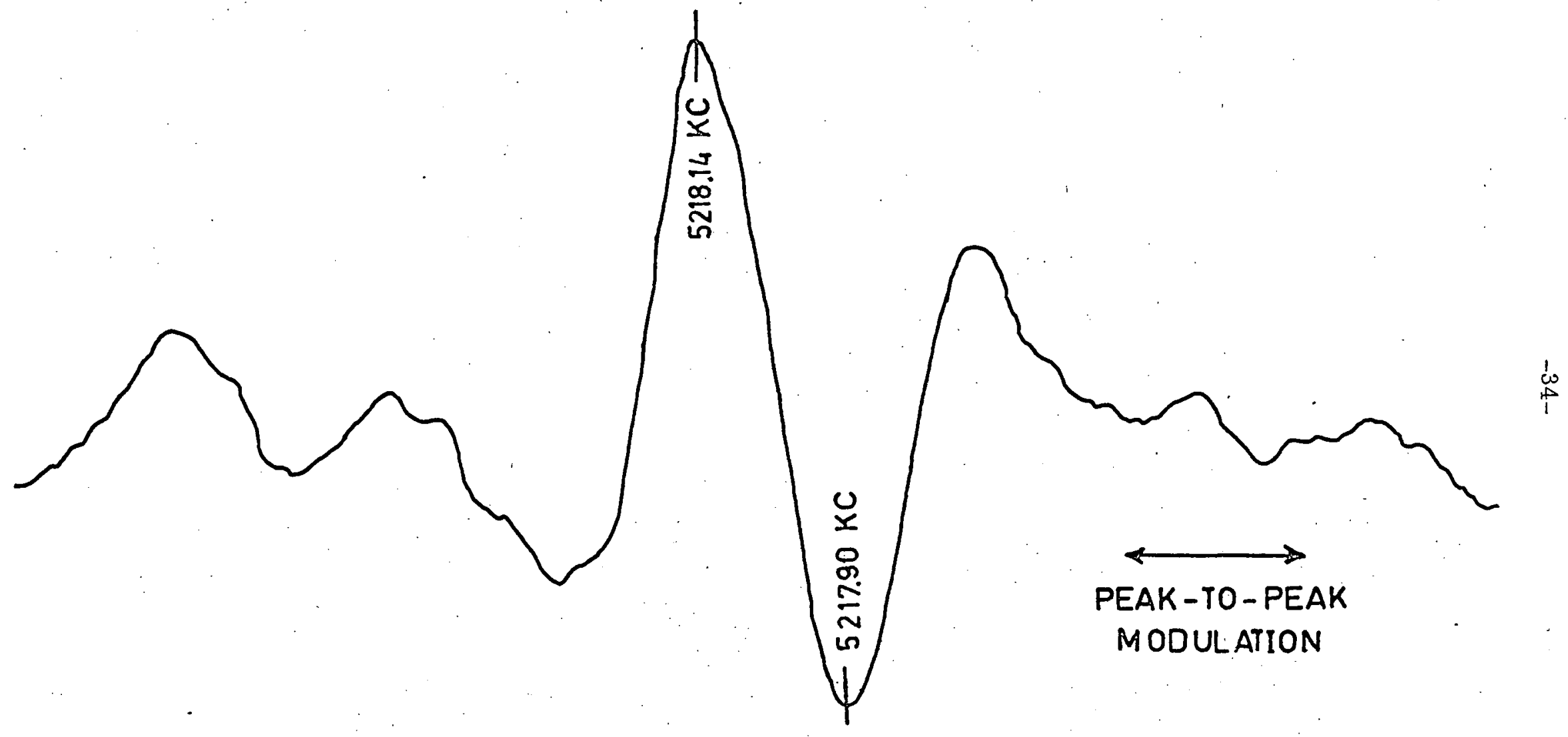

Fig. 7 Resonance Line Corresponding to the $\left(\frac{1}{2} \rightarrow-\frac{1}{2}\right)$ Transition of $\mathrm{Mg}^{25}$ in a Single Crystal 
and at $1.2^{\circ} \mathrm{K}$ was badly distorted by saturation effects, a long spinlattice relaxation time is possibly indicated. The signal-to-noise ratio was too small at liquid nitrogen temperature to provide a distinguishable Iine.

The satellite lines from the quadrupole splitting could not be observed. This is not surprizing in view of the small signal from the more intense central line and the fact that the width and intensity of satellite lines are very sensitive to the effects of strains and imperfections in the lattice.

The line is difficult to recognize as a second derivative signal because of the broadening and distorting effects mentioned in the previous chapter. This undesirable distortion did not affect the accuracy of the measurements greatly because of the relatively narrow line width of about $230 \mathrm{~Hz}$. The résonance frequency of the line was determined by comparing the line shape with overmodulated line shapes calculated theoretically and applying a correction to account for the mixture of absorption and dispersion modes. This correction could affect only the accuracy of the parameter $\mathrm{K}_{\text {iso }}$, so an accordingly larger uncertainty in its value has been attached to it. Since the line width and shape did not vary significantly with the angle of the field, any convenient reference point on the line would suffice to determine the anisotropic part $K^{\prime}$ and the quadrupole splitting without a correction being necessary. 
Figure 8 shows the orientational dependence of the resonance frequency of the centre line. The experimental points were fitted to the expression of equation (5I) by a least squares fit routine offered by the U.B.C. Computing.Centre. With the experimental points is shown the resulting fitted curve which yields the parameters given in the table below. The uncertainties attached to the values

\begin{tabular}{|c|c|c|c|c|c|}
\hline & $\mathrm{K}$ & $\mathrm{K}^{\prime}$ & $\mathrm{e}^{2} \mathrm{qQ} / \mathrm{h}(\mathrm{exp})$ & $\mathrm{e}^{2} \mathrm{q}_{\mathrm{ion}} \mathrm{Q} / \mathrm{h}$ (calc) & $\mathrm{c} / \mathrm{a}$ \\
& $\%$ & $\%$ & $\mathrm{KHz}$ & $\mathrm{KHz}$ & \\
\hline Rm. Temp. & $0.111^{\mathrm{a}}$ & & $230^{\mathrm{a}}$ & 93 & 1.6235 \\
$4.2^{\mathrm{o}} \mathrm{K}$ & $0.1127 \pm 0.0005$ & $0.0004 \pm 0.0002$ & $324 \pm 6$ & 100 & 1.6230 \\
\hline
\end{tabular}

a see reference 17

are the statistical root mean square deviations supplied by the computer program.

The table compares the results with those of Rowland $(17)$ for powdered magnesium at room temperature. The calculated value for the coupling constant was arrived at by use of equations (46) and (47)

$$
q_{\text {lattice }}=\sum_{i} \frac{3 z_{i}^{2}-x_{i}^{2}}{r^{5}} ; q_{i o n}=z\left(1+\gamma_{\infty}\right) q_{\text {lattice }}
$$

The summation was carried out directly over 400,000 sites and the same results were obtained with an expression by Das and Pomerantz, (18). 
Fig. 8 Measured Frequency Shift of Central Component of the $\mathrm{Mg}^{25}$ Resonance

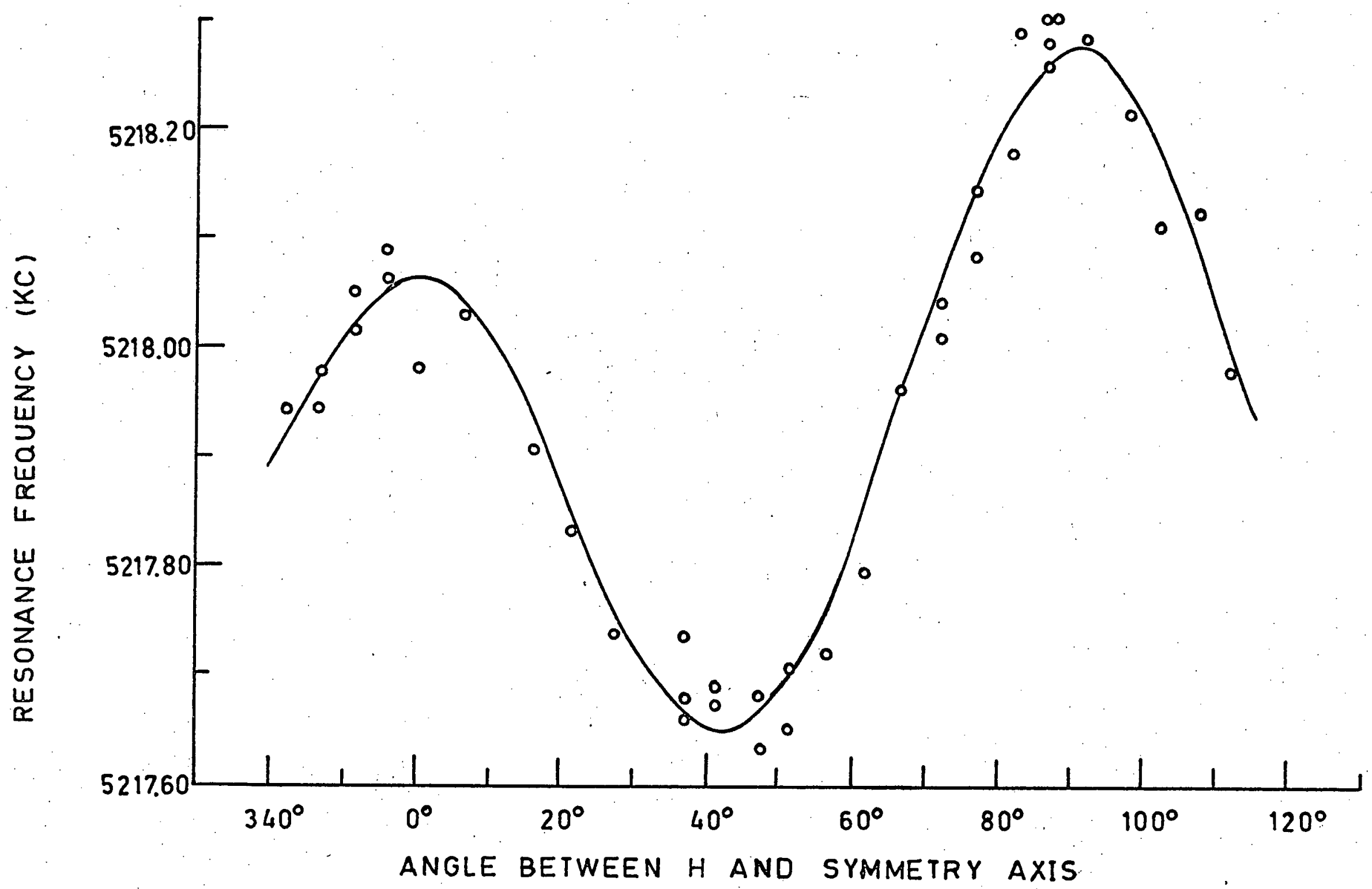


$q_{\text {lattice }}=[0.0065-4.3584(\mathrm{c} / \mathrm{a}-1.633)] / \mathrm{a}^{3}$. These were evaluated at both temperatures using the coefficients of expansion for magnesium. For $q_{i o n}$ a normal valence of 2 was assumed and $\gamma_{\infty}$, the antishielding factor used, was 3.2. ${ }^{(20)}$ The value of the electric quadrupole moment, $Q=0.22 \times 10^{-24} \mathrm{~cm}^{2}$, is from nuclear beam data. ${ }^{(21)}$

\section{B. Comparison with Powder Data}

Before comparing the quadrupole coupling constant to Rowland's value some characteristics of powdered line shapes will' be discussed.

In single crystal magnesium, the frequency of the central transition is given by

$$
\nu_{\frac{1}{2}}=\nu_{0}+\frac{\nu_{Q}^{2}}{2} \nu_{0}\left(\mu^{2}-1\right)\left(9 \mu^{2}-1\right)+a\left(3 \mu^{2}-1\right)
$$

as in equation (51) but with $\mu=\cos \theta, a=K^{\prime} / 2$, and $I=5 / 2$. To deduce the line shape from a polycrystalline sample, consider $\mathrm{P}\left(\nu-\nu_{0}\right) \mathrm{d}\left(\nu-\nu_{0}\right),{ }^{(11)}$ the probability that $\left(\nu-\nu_{0}\right)$ lies between $\left(\mathcal{V}-\mathcal{\nu}_{0}\right)$ and $\left(\mathcal{\nu}-\mathcal{\nu}_{0}\right)+d\left(\nu-\mathcal{\nu}_{0}\right)$. Since the probability that a particular crystallite has its $z$ axis at an angle to the magnetic field is proportional to $\sin \theta$, we have

$$
\mathrm{P}\left(\nu-\nu_{0}\right) \mathrm{d}\left(\nu-\nu_{0}\right)=\mathrm{P}(\theta) \mathrm{d}(\theta)=\frac{1}{2} \sin \theta \mathrm{d} \theta=\frac{1}{2} \mathrm{~d} \mu
$$

so that $\mathrm{P}\left(\nu-\nu_{0}\right)=\frac{1}{2} \mathrm{~d} \nu / \mathrm{d} \mu^{-1}$ for $-1 \leq \mu \leq 1$; the factor $\frac{1}{2}$ occurs because $+\theta$ and $-\theta$ correspond to the same frequency. Now from (52)

$$
\mathrm{d}\left(\nu-\nu_{\mathrm{o}}\right) \cdot \mathrm{d} \mu=\left(2 \nu_{\mathrm{Q}}^{2} / \nu_{\mathrm{o}}\right) \mu\left[\left(9 \mu^{2}-5\right)+3 \nu_{\mathrm{o}}^{2} \mathrm{a} / \nu_{\mathrm{Q}}^{2}\right]
$$


This shape function has singularities occurring at $\mu=0$ and $\mu=\mu^{\prime}=$ $\pm\left[5 / 9-a \nu_{0}^{2} / 3 \nu_{Q}^{2}\right]^{\frac{1}{2}}$. These $\mu^{\prime}$ are not distinguishable since $\nu-\nu_{0}$ depends only on $\mu^{2}$. Besides the singularities a discontinuity occurs in the shape function at $\mu=1$. The line shape arising from this treatment is in figure 9. Rowland attributed the splitting of the central transition to the quadrupole interaction alone, neglecting any possible contribution from Knight shift anisotropy (i.e., $a=0$ in the above). This would produce the line shape of figure 9a using his value, $e^{2} \mathrm{qQ} / \mathrm{h}=230 \mathrm{KHz}$. If our value measured at $4.2^{\circ} \mathrm{K}, \mathrm{e}^{2} \mathrm{qQ} / \mathrm{h}=$ $324 \mathrm{KHz}$, is used with no anisotropy, the resulting line would be as in figure $9 \mathrm{~b}$. If our value holds at room temperature, an anisotropy of $0.056 \%$ would be necessary in addition to the quadrupole effect to produce the same splitting Rowland observed. Since it is the derivate of the line which is detected, line 9 a would not be distinguishable from Iine $9 \mathrm{c}$ in a system with a poor signal-to-noise ratio. This increase from $0.0004 \%$ at $4.2{ }^{\circ} \mathrm{K}$ to $0.056 \%$ at room temperature would be a surprizing result. The analysis of line $9 \mathrm{c}$ also affects the position of $\mathcal{V}_{\mathrm{o}}$ as can be seen, and would require a $10 \%$ increase. in $\mathrm{K}_{\text {iso }}$.

Alternatively, if this large change in anisotropy does not occur, a highly temperature dependent quadrupole interaction would produce an agreement with Rowland's results. Of course, any suitable combination of the temperature dependences of the two effects would also resolve the discrepancy. More recently, Drain ${ }^{(22)}$ has reported an anisotropy too smail. to measure at room temperature in powdered 
FIGURE 9 THEORETICAL POWDER SIGNAL SHAPES

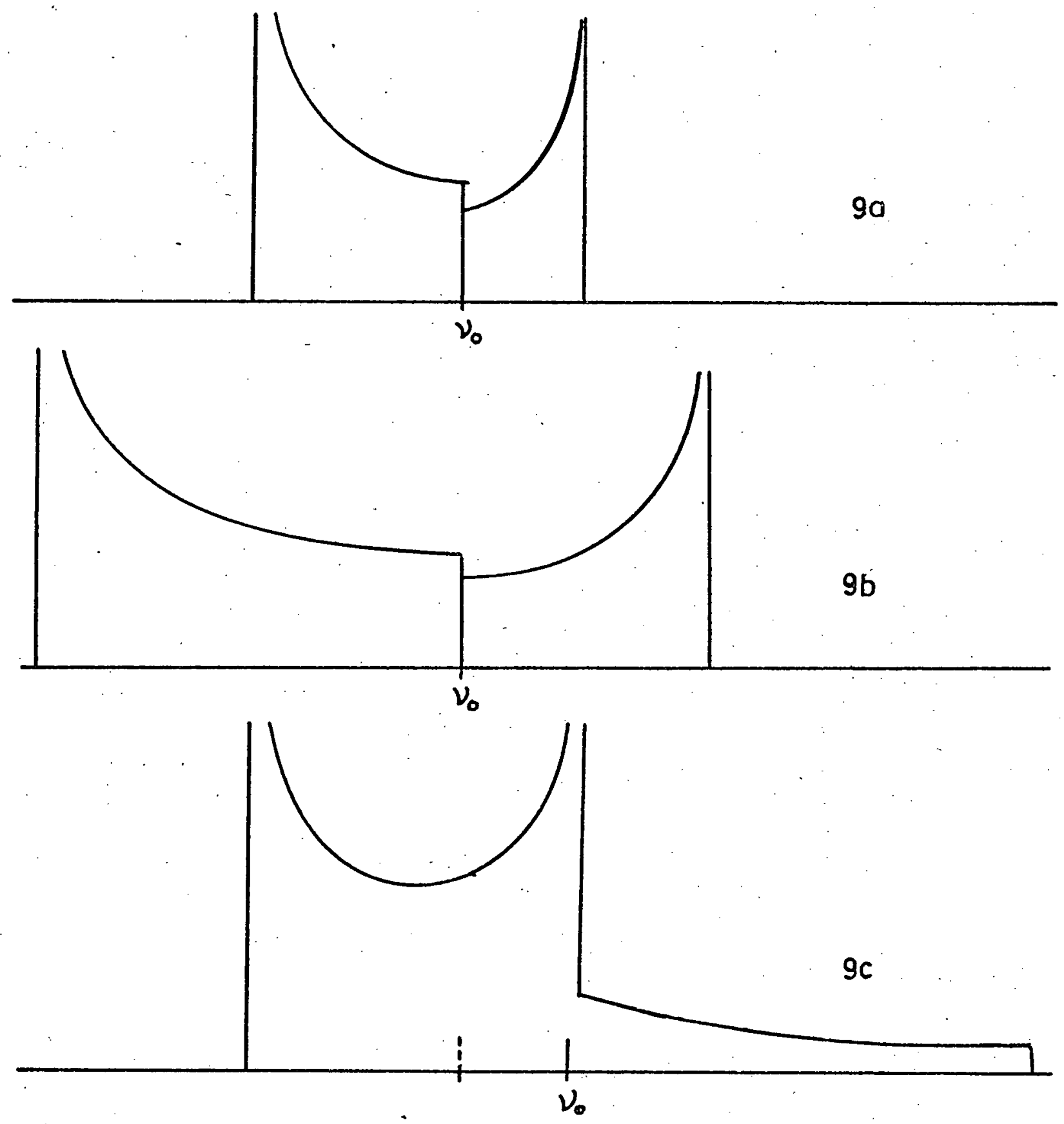

Fig. 9a Rowlands value ${ }^{d}$ eq ${ }^{2} \mathrm{Q} / \mathrm{h}=230 \mathrm{KHz}$ with $\mathrm{K}^{\prime}=0$

Fig. 9b Present value $\left(4.2^{\circ} \mathrm{K}\right), \mathrm{eq}^{2} \mathrm{Q} / \mathrm{h}=324 \mathrm{KHz}$ with $\mathrm{K}^{\prime}=0$

Fig. 9c eq ${ }^{2} \mathrm{Q} / \mathrm{h}=324 \mathrm{KHz}$ with $\mathrm{K}^{\prime}$ adjusted to give. same signal width Rowland observed

$\mathrm{d}_{\text {See }}$ reference 17 
magnesium. Although no limits are given, we can assume that the anisotropy was not large enough to effect his deduced coupling constant which agrees with Rowland's. This would indicate that it is the coupling constant, $\mathrm{e}^{2} \mathrm{qQ} / \mathrm{h}$, which undergoes a large temperature variation. As shown, a single measurement in powder can lead to ambiguous results, especially where weak signals are investigated. However, since the quadrupole splitting varies inversely with $\nu_{0}$ and the splitting due to the anisotropy varies directly with $\nu_{0}$, it is possible to separate the contributions of each by measuring the splitting as a function of the applied field. (14)

\section{Role of the Conduction Electrons}

Measuring the coupling constant $\mathrm{e}^{2} \mathrm{qQ} / \mathrm{h}$, gives an experimental value for eq, the field gradient parameter. We also have, from chapter 2 , a calculated value,

$$
q_{\text {ion }}=z\left(1+\gamma_{\infty}\right) q_{\text {latt }}
$$

the parameter obtained by considering the ionic model of the metal. Temperature, through thermal expansion altering the lattice parameters, does effect the lattice contribution to the electric field gradient, though an evaluation of $\mathrm{q}_{\text {lattice }}$ at different temperatures shows for magnesium that the effect is only $7 \%$. from 300 to $4.2^{\circ} \mathrm{K}$. By comparing the experimental and calculated values (see Table 1 ) it is evident that $q_{i o n}$ accounts for only part of the field gradient at either 
temperature. The remainder must be attributed to the electric field gradient, called $\mathrm{q}_{\text {cond }}$, arising from a non-spherical distribution of the conduction ejectrons. We see that $q_{\text {cond }} q_{\text {ion }}$ and seems to increase with decreasing temperature from about $2.5 \mathrm{q}_{\text {ion }}$ at room temperature to about $3.2 \mathrm{q}_{\text {ion }}$ at $4.2^{\circ} \mathrm{K}$. $\mathrm{q}_{\text {cond }}$ appears to be positive but since this experiment does not determine the sign of $q$, we can make statements concerning only magnitudes.

$$
\text { An accurate evaluation of } q_{\text {cond }}=\left\langle\psi\left|3 \cos ^{2} \theta-1\right| \psi\right\rangle \text {, }
$$

the quadrupole moment of the conduction electrons gives a measure of their non-spherical nature and their contribution to their field gradient. This requires a knowledge of the orbital characteristics of all the conduction states in the metal, whereas for the anisotropic Knight shift, $q_{F}=\left\langle\psi\left|3 \cos ^{2} \theta-1\right| \psi\right\rangle_{F}$ (eqn. 14), required only the states near the Fermi level.

To calculate their effect on the field gradient, it is sometimes useful to consider the conduction electrons with a sphere centered about the nucleus. If the density within this sphere is spherically symmetric, then the nucleus at the centre will feel no additional field gradient but the other nuclei in the lattice may experience a different $q_{\text {lattice }}$ because of the effectively different charge at the lattice site. This additional charge may be positive or, as in the case of Berylium, (23) negative depending on whether a net electron or a net hole density is considered. This may account partially for the fact that $q>q_{i o n}$. 
Watson, Gossard and Yafet ${ }^{(24)}$ suggest that $q_{\text {cond }}$ may be separated into 3 terms,

$$
q_{\text {cond }}=q^{0}+q^{\prime}+q^{\prime \prime}
$$

where $\mathrm{q}^{\circ}$ is the local gradient obtained by constructing the Fermi surface on the assumption the Block functions are eigenfunctions of a spherical potential within the atomic sphere. The $q^{\prime}$ contribution arises from the external environment producing a potential within the atomic sphere which causes a first-order shift in the Block function energies, bringing about a redistribution of occupied states in the vicinity of the Fermi surface. The term $q^{\text {it }}$ is associated with the spatial distortion of the Block functions.

The terms $\mathrm{q}^{\circ}$ and $\mathrm{q}^{\mathrm{q}}$ are dependent on the behavior of the conduction states below the Fermi energy $E_{F}$, but it is suggested that the dominant contribution, that of $q^{\prime}$, is strongly dependent on $N\left(E_{F}\right)$ the density of states near the Fermi surface only. The term $q^{\prime}$ is opposite in sign to $\mathrm{q}_{\text {latt}}$, therefore shielding it, and in some cases (transition and some p-band metals) the ratio $q 1 / q_{\text {lattice }}$ may be as high as 100. Since it is also shown that the Fermi-Dirac distribution introduces a large temperature dependence of $q^{\prime}$, the total $q$ may well be determined by $q^{\prime}$ and exhibit a significant temperature variation. Although the density of states has a significant variation with temperature only for metals with narrow conduction bands (d-band in transition metals) and does not vary significantly for s electrons, this mechanism may account in part for the temperature dependence observed in magnesium. 
CHAPTER V

CONCLUSIONS

\section{A. Comparison with Cadmium}

Both the isotropic and anisotropic shifts in cadmium vary markedly with temperature. $(2,3,4)$ The same behavior apparently is not repeated in magnesium; this is probably due to the difference between the $\mathrm{c} / \mathrm{a}$ ratios in the two metals and to the relatively simpler band structure of magnesium.

The large temperature dependence of $\mathrm{K}_{\text {anis }}$ in cadmium suggests that the quadrupole moment of the electrons may be related to the change in the c/a ratio due to the anisotropic thermal expansion. (25) A decrease in temperature from $300^{\circ} \mathrm{K}$ to $4.2^{\circ} \mathrm{K}$ is accompanied by a decrease in $\mathrm{c} / \mathrm{a}$ from 1.8857 to 1.8640 , a change of $1.2 \%$. Within this same temperature range, $\mathrm{K}_{\text {anis }}$ decreases from $5.0 \times 10^{-4}$ through 0.0 at about $60^{\circ} \mathrm{K}$, to $-1.0 \times 10^{-4}$ at $4.2^{\circ} \mathrm{K}$. (15) The speculation is that the electron distribution becomes spherical at some particular c/a ratio. To investigate this possible dependence Borsa and Barnes ${ }^{(2)}$ considered the effect of alloying magnesium with cadmium. The presence of 1.0 atomic wt. \% $\mathrm{Mg}$ in $\mathrm{Cd}$ achieved about the same change in $\mathrm{c} / \mathrm{a}$ as lowering the temperature of pure $\mathrm{Cd}$. They observed no significant change in the parameter. It is not apparent that conclusions drawn from this method are valid since other effects of alloying are not sufficiently understood. Cadmium does, however, exhibit a large 
temperature dependence in the anisotropy of the diamagnetic susceptibility. This also decreases greatly in the same temperature range but its relation to the Knight shift awaits explanation.

The results from magnesium, though revealing a $K$ anis which is perhaps too small to enable one to draw valid conclusions, tends to support the idea of a c/a dependence. From $300^{\circ} \mathrm{K}$ to $4.2^{\circ} \mathrm{K}$, magnesium undergoes a change in c/a from 1.6235 to 1.6230, a decrease of only $0.03 \%$, while displaying no significant change in $\mathrm{K}_{\text {anis }}$ (see Table 1). The small (if any) variation is consistant with a model built up of perfectly spherical atoms. Such a lattice would have a c/a ratio of 1.6333, very close to magnesium's 1.6235, and would not be expected to display anisotropic features.

The temperature dependences of the parameters in cadmium may be primarily due to the electron wave functions adjusting themselves to accommodate the rapid changes of volume occupied by an atom as it undergoes lattice vibrations. These variations in the wavefunctions would manifest themselves more in cadmium than in magnesium because of the difference in complexity of their Fermi surfaces.

The temperature variation in the quadrupole coupling constant would reflect a change in $\left\langle\psi\left|3 \cos ^{2} \theta-1\right| \psi\right\rangle_{F}$, the electron quadrupole moment at the Fermi surface, if the theory of Watson, Gosard and Yafet is applicable. Since this would suggest an accompanying variation in $\mathrm{K}_{\text {anis }}$ which was not observed, two conclusions may be drawn; either a substantial contribution to $q$ arises from the electron 
states well below the Fermi level, or the states at the surfaces are anisotropic but their contribution is nullified by a core polarization contribution.

\section{B. Further Studies}

No strong statements can be made about the temperature dependence on the basis of measurements at only two temperatures. It would be desirable to continue this study in magnesium for various temperatures. Unless signals from the single crystal can be improved, this would necessarily be done in a powdered sample with subsequent loss of resolution.

The next candidate for study in this system of bivalent

hexagonal close-packed metals is zinc. $\mathrm{Zn}^{67}$, with a low natural abundance (4.2\%) and large quadrupole interaction (estimated coupling constant as high as $70 \mathrm{MHz}(27)$, would, however, provide a very small, perhaps undetectable, signal.

These present measurements on magnesium should provide an interesting experimental comparison when a detailed theoretical treatment is available. This seems likely in view of the present detailed knowledge of its band structure ${ }^{(28)}$ and its comparatively Iow atomic number. 


\section{BIBLIOGRAPHY}

(1) F.J. Milford, Am. J. Phys. 28, 521 (1960).

(2) F. Borsa and R.G. Barnes, J. Phys. Chem. of Solids 27, 567 (1966).

(3) E.F.W. Seymour and G.A. Styles, Physics Letters, 10, 269 (1964).

(4) H.E. Schone, Phys. Rev. Letters 13, 12 (1964).

(5) C.P. Slichter, "Principles of Magnetic Resonance", Harper and Row, p. 97 (1963).

(6) N. Bloembergen and T.J. Rowland, Acta Met. I, 731 (1953).

(7) V.I. Checkernikov, I. Pop, and 0.P. Naumkin, Sov. Phys. - JETP I7, 1228: (1963).

(8) N.F. Ramsay, "Nuclear Moments", Willey, p. 23 (1953).

(9) A. Abragam, "The Principles of Nuclear Magnetism", Oxford University Press, p. 233 (1961).

(10) H.E. Petch, N.G. Cranna and G.M. Volkoff, Can. J. Phys. 31, 837 (1953).

(11) M.H. Gohen and F. Reif, Solid State Phys. ㅁ, 321 (1957).

(12) G.E. Pake, Solid State Phys. 2, I (1956).

(13) A.C. Chapman, P. Rhodes and E.F.W. Seymour, Proc. Phys. Soc. 70, B 345 (1957).

(14) W.H. Jones, Jr., T.P. Graham and R.G. Barnes, Phys. Rev. I32, 1898: (1963).

(15) S.N. Sharma, Ph.D. Thesis, University of British Columbia (1967).

(16) G.V.H. Wilson, J. App. Phys. 34, 3276 (1963).

(17) T.J. Rowland, Nuclear Magnetic Resonance in Metals, Progress in Materials Science, vol. 9 (1961).

(18) T.P. Das and M. Pomerantz, Phys. Rev. I23, 2070 (196I).

(19) E. Goens and E. Schmid, Phys. Z. 37, 385 '(1936); R.M. McCammon and G.K. White, Phil. Mag. 11, $11 \overline{25}$ (1965).

(20) G. Burns, J. Chem. Phys. 3I, 1253 (1959).

(21) A. Lurio, Phys. Rev. 126, 1768 (1962). 
(22) L.E. Drain, Metallurgical Reviews, Vol. 12, p. 195 (1967).

(23) M. Pomerantz and T.P. Das, Phys. Rev. 1ㅡ, 70 (1960).

(24) R.E. Watson, A.C. Gosard and Y. Yafet, Phys. Rev. 140 , A375 (1965).

(25) .E. Griieisen and E. Goens, Z. Physik, 29, 141 (1924).

(26) J.A. Marcus, Phys. Rev. 76, 621 (1949).

(27) G. Seidel and P.H. Keesom, Phys. Rev. Lett. 2, 261 (1959).

(28) L.M. Falicov, Phil. Trans. Roy. A255, 55 (1962). 